\title{
Reconstructed Phase Space-Based Damage Detection Using a Single Sensor for Beam-Like Structure Subjected to a Moving Mass
}

\author{
Zhenhua Nie, ${ }^{1,2}$ Tuan Ngo, ${ }^{3}$ and Hongwei $\mathrm{Ma}^{1,4,5}$ \\ ${ }^{1}$ College of Science \& Engineering, Jinan University, Guangzhou, Guangdong, China \\ ${ }^{2}$ State Key Laboratory for Manufacturing Systems Engineering, Xi'an Jiaotong University, Xi'an, Shanxi, China \\ ${ }^{3}$ Department of Infrastructure Engineering, The University of Melbourne, Melbourne, VIC, Australia \\ ${ }^{4}$ Dongguan University of Technology, Dongguan 523000, China \\ ${ }^{5}$ College of Civil Engineering, Qinghai University, Xining, China
}

Correspondence should be addressed to Hongwei Ma; tmahw@jnu.edu.cn

Received 17 June 2016; Revised 31 October 2016; Accepted 24 November 2016; Published 28 February 2017

Academic Editor: Ivo Caliò

Copyright (C) 2017 Zhenhua Nie et al. This is an open access article distributed under the Creative Commons Attribution License, which permits unrestricted use, distribution, and reproduction in any medium, provided the original work is properly cited.

\begin{abstract}
This paper presents a novel damage detection method based on the reconstructed phase space of vibration signals using a single sensor. In this approach, a moving mass is applied as excitation source, and the structure vibration responses at different positions are measured using a single sensor. A Moving Filter Function (MFF) is also presented to be used to separate and filter the responses before phase space reconstruction. Using the determined time delay and embedding dimensions, the responses are translated from time domain into the spatial domain. The index CPST (changes of phase space topology) values are calculated from the reconstructed phase space and used to identify structural damage. To demonstrate the method, six analysis scenarios for a beamlike structure considering the moving mass magnitude, damage location, the single sensor location, moving mass velocity, multiple types of damage, and the responses contaminated with noise are calculated. The acceleration and displacement responses are both used to identify the damage. The results indicate that the proposed method using displacement response is more sensitive to damage than that of acceleration responses. The results also proved that the proposed method can use a single sensor installed at different location of the beam to locate the damage/much damage reliably, even though the responses are contaminated with noise.
\end{abstract}

\section{Introduction}

Structural Health Monitoring (SHM) is a practical tool in the prediction of safety level and system performance of in-service structures, while damage detection is an important task in SHM and condition assessment of structures. Vibration-based damage detection methods are based on the fact that any change introduced in a structure results in changes in its dynamic behavour. Thus, theoretically, occurrence of even a small damage will change the physical characteristics of a structure (its mass, stiffness, and damping characteristics), which in turn will affect its vibration response and change its dynamic characteristics. Vibrationbased damage detection methods are especially attractive because they are global monitoring methods in the sense that none a priori information for the location of the damage is needed and/or immediate access to the damaged part is not required for structural damage detection [1].

The most commonly used vibration parameters for structural damage detection are frequencies $[2,3]$, mode shapes, and mode shape related parameters such as MAC, COMAC [4], flexibility [5], mode shape derivatives [6-8], and frequency response functions (FRF) based parameters [9]. An overview of the vibration-based methods for structural damage detection can be found in $[10,11]$. Although the theory of vibration-based methods is straightforward and are generally accepted for the purposes of structural damage detection, they still pose a number of problems and challenges in practical application, such as the low sensitivity, relying on an accurate structure model, and so on. Particularly, modal based 
methods need enough sensors to insure the accuracy of the mode. The future development direction of structural health monitoring is how to deal with the real engineering and solve the specific problems. At present, an important reason that the health monitoring technology is difficult to be applied in real engineering is that it requires a large number of sensors, as well as the transmission, recording, and processing equipment. It is impractical and uneconomical to install sensors on the whole structure in all degrees of freedom. Therefore, we must develop the methods using less sensors to solve this problem.

In recent years, many researchers have sought to use a small amount of sensors, even a single, to detect structural damage, which becomes a hot issue in the research. The typical method is applying a moving load (vehicle load in bridge structure) as excitation source, and using a single sensor to measure the structure response at a certain position to identify the damage location. This approach is closely related to the practical engineering, does not find the problem of optimizing the allocation of sensors, and is a qualitative leap in the sensor consumption. This idea was put forward early in 2006 by Zhu and Law [12]. The authors proposed a method using the response obtained at a single measuring point of a beam structure and analyzed by Continuous Wavelet Transform (CWT) and the location of the cracks was estimated. The locations of the cracks were determined from the sudden changes in the spatial variation of the transform responses. Hester and González [13] using the acceleration signal and employing a vehicle-bridge finite element interaction model developed a wavelet based approach using wavelet energy content at each bridge section, which was proved to be more sensitive to damage than a wavelet coefficient. Nguyen and Tran [14] proposed a method that the dynamic response of the bridge-vehicle system measured directly from the moving vehicle was analyzed by wavelet transform. The locations of the cracks were pinpointed by positions of peaks of the transform responses. Khorram et al. [15] compared two wavelet based damage detection approaches to find the location and the size of a crack in a beam subjected to a moving load, one of which uses the time varying deflection attributed to the beam at midspan, while in another the sensor is attached to the moving load. It is found that the moving sensor approach is more effective than the fixed sensor. Later, Khorram et al. [16] proposed another method of multiple cracks detection in a simply supported beam subjected to a moving load based on CWT combined with factorial design. The similar wavelet based methods can be found in $[17,18]$.

In addition to the methods of wavelet transform, in 2012, Roveri and Carcaterra [19] proposed a novel HHTbased method for damage detection of bridge structures under a travelling load. The technique uses a single point measurement and is able to identify the presence and the location of the damage along the beam. The measured data is processed by the HHT technique. And none a priori information is needed about the response of the undamaged structure. Damage location is revealed by direct inspection of the first instantaneous frequency, which presents a sharp crest in correspondence with the damaged section. The advantage of this method is that it needs no baseline data, but it shows no sensitivity enough as the crack cannot be identified when the crack depth ratio is $20 \%$. Li and Law [20] presented a substructural damage identification approach under moving vehicular loads based on a dynamic response reconstruction technique. The effectiveness of this method was proved by an experiment [21]. Cavadas et al. [22] presented a damage detection method using data-driven methods applied to moving load responses, which focuses on two data-driven methods: moving principal component analysis (MPCA) and robust regression analysis (RRA). But to locate the damage, the proposed method also needs a large amount of data measured by enough sensors. Zhang et al. [23] presented a local damage detection method for beam and plate like structures based on operating deflection shape curvature extracted from dynamic response of a passing vehicle.

Actually, to the best of authors' knowledge, the articles with the idea that using the response measured with single sensor to locate structural damage are relatively rare in SHM (generally the above-mentioned methods). All these approaches have something in common; the damage is visualised through the appearance of a singularity in a processed signal supposed to be smooth in a healthy case. This paper focuses on using the phase space reconstructed method to detect the damage with single sensor. Phase space approach is a novel signal preceding method which is proved sensitive to damage [24-28]. This approach transfers the response from time domain into the spatial domain to analyze the vibration properties. Dynamics are most easily understood when viewed from a phase space perspective. Therefore, phase space-based classification for the response of a structural system is employed for structural damage detection because the effect of damage alters the behavior of phase trajectory [29-31]. In the previous study, Nie et al. [32] proved that the damage index extracted from reconstructed phase space using ambient excitation is significantly more sensitive to damage than modal based methods but relatively insensitive to noise and used this index in the experimental study of a two-span RC slab (6 m length) for damage detection, which proved that it is a good candidate for continuous structural health monitoring [1]. This approach also requires enough number of sensors to locate the damage. However, Nie et al. [33] also proved that the reconstructed phase space of the response measured at a single point of the beam, compared to the undamaged condition, would warp during the time of moving load passing by the damage zone. This phenomenon indicates that reconstructed phase space can be used as a candidate for damage localization using a single sensor approach.

To the best of authors' knowledge, all the methods use a single point mass/load to generate the vibration data and, there are no literature studies on the application of phase space-based techniques for the health monitoring of civil structures under moving mass/load. Therefore, an innovative technique, phase space-based using a single sensor, for damage detection of a bridge structure excited by a moving mass is here proposed, and the damage index extracted from the response phase space is presented. 


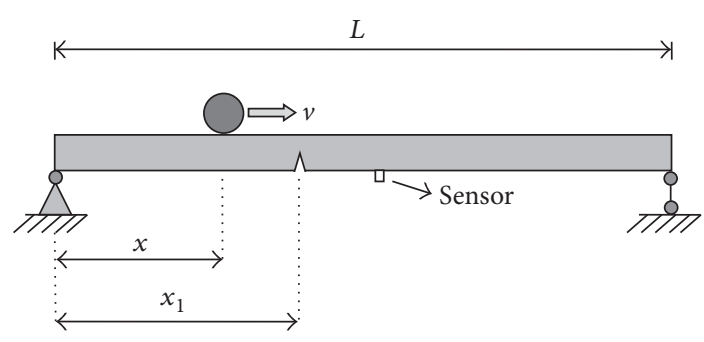

FIGURE 1: Sketch of the beam model subject to a moving mass.

\section{Dynamic Theory of Simple Beam Subject to a Moving Mass}

The uniform Euler-Bernoulli beam with moving mass is considered as shown in Figure 1. A moving mass $m_{m}$ travels along the beam with a constant velocity $v$. The bending stiffness, $E I$ and the mass per unit length of the beam $m$, is assumed to be constant. Let $w(x, t)$ denote the deflection of the beam with $x$ and $t$ representing the position of a point in the beam and the time $(t=0$ as the moving mass enters the beam from left to right), respectively. For a one-dimensional beam problem, the position vector $x$ will represent the $x$-axis with its origin coincided on the left support. The governing differential equation for vibration of the beam within the domain $x \in[0, L]$, neglecting the damping, the rotary inertia, and shearing force effects can be written as

$$
m(x) \frac{d^{2} w(x, t)}{d t^{2}}+D w(x, t)=p(x, t),
$$

where

$$
\begin{gathered}
D=\frac{\partial^{2}}{\partial x^{2}}\left[E I \frac{\partial^{2}}{\partial x^{2}}\right]=E I \frac{\partial^{4}}{\partial x^{4}}, \\
p(x, t)=m_{m}\left(g-\frac{d^{2} w(x, t)}{d t^{2}}\right) \delta(x-v t) .
\end{gathered}
$$

The term $p(x, t)$ denotes the interaction force between moving mass and beam (assuming continuous contact between the mass and beam), and $g$ is the gravitational acceleration and $\delta(x-v t)$ is Dirac delta function that represents the location of the moving mass with the speed of $v$ at time $t$. In (3), the differential term can be expanded as

$$
\frac{d^{2} w(x, t)}{d t^{2}}=\frac{\partial^{2} w(x, t)}{\partial t^{2}}+2 v \frac{\partial^{2} w(x, t)}{\partial x \partial t}+v^{2} \frac{\partial^{2} w(x, t)}{\partial x^{2}}
$$

Hence (3) can be rewritten as

$$
\begin{aligned}
& p(x, t)=m_{m}\left(g-\frac{\partial^{2} w(x, t)}{\partial t^{2}}-2 v \frac{\partial^{2} w(x, t)}{\partial x \partial t}\right. \\
& \left.-v^{2} \frac{\partial^{2} w(x, t)}{\partial x^{2}}\right)_{x=v t} \delta(x-v t) .
\end{aligned}
$$

Using the eigenfunction expansion, deflection $w(x, t)$ can be considered as

$$
w(x, t)=\sum_{i=1}^{n} \phi_{i}(x) q_{i}(t)
$$

where $\phi_{i}(x)$ is the $i$ th orthogonal modal shape of the beam, $q_{i}$ is time-dependent modal amplitudes function, and $n$ denotes the total number of considered modes. Substituting above expression in (5), the result is

$$
\begin{aligned}
& p(x, t)=m_{m}\left(g-\sum_{i=1}^{n}\left[\phi_{i}(x) \frac{\partial^{2} q_{i}(t)}{\partial t^{2}}\right.\right. \\
& \left.\left.+2 v \frac{\partial \phi_{i}(x)}{\partial x} \frac{\partial q_{i}(t)}{\partial t}+v^{2} \frac{\partial^{2} \phi_{i}(x)}{\partial x^{2}} q_{i}(t)\right]\right) \\
& \quad \delta(x-v t) .
\end{aligned}
$$

Substituting (7) and (6) into the beam equation of motion (1), multiplying by $\phi_{k}(x)$, and integrating for the entire beam yield

$$
\begin{aligned}
& \int_{0}^{L} m(x) \sum_{i=1}^{n} \phi_{i}(x) \phi_{k}(x) \ddot{q}_{i}(t)+m(x) \omega_{i}^{2} \sum_{i=1}^{n} \phi_{k}(x) \\
& \cdot q_{i}(t) d x=\int_{0}^{L} m_{m}(g \\
& \left.-\sum_{i=1}^{n}\left[\phi_{i}(x) \ddot{q}_{i}(t)+2 v \phi_{i}^{\prime}(x) \dot{q}_{i}(t)+v^{2} \phi_{i}^{\prime \prime}(x) q_{i}(t)\right]\right) \\
& \cdot \phi_{k}(x) \delta(x-v t) d x
\end{aligned}
$$

where

$$
m(x) \omega_{i}^{2}=D \phi_{i}(x)
$$

Using the orthogonality condition of eigenfunctions and rearranging (8), the results are

$$
\ddot{q}_{i}+\omega_{i}^{2} q_{i}=Q_{i},
$$

where

$$
\begin{aligned}
Q_{i} & =\frac{m_{m}}{m(x)} \phi_{i}(v t)\left[g-\sum_{k=1}^{n} \ddot{q}_{k} \phi_{k}(v t)-2 v \sum_{k=1}^{n} \dot{q}_{k} \phi_{k}^{\prime}(v t)\right. \\
& \left.-v^{2} \sum_{k=1}^{n} q_{k} \phi_{k}^{\prime \prime}(v t)\right] .
\end{aligned}
$$

In case moving force with the coupling vibration between the mass and bridge is neglected (see (5)), the operator $d^{2} w(x, t) / d t^{2}$ is equal to zero and one should only consider the magnitude of the moving force. In a similar study of a single damaged beam under a moving mass [34] the convective terms Eq. (5) were omitted. However, this approximation is not generally reasonable unless the mass moves at a very low speed, and it may lead to significant errors in the evaluation of the system response. In (5), the term $2 v\left(\partial^{2} w(x\right.$, $t) / \partial x \partial t)$ is derived from Coriolis force and plays the role of damping. However the term $v^{2}\left(\partial^{2} w(x, t) / \partial x^{2}\right)$ is derived from centrifugal force and almost plays the role of weakening the bending stiffness of the beam. Also, the term $\partial^{2} w(x, t) / \partial t^{2}$ 


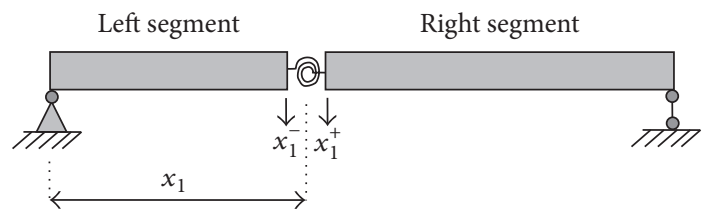

Figure 2: Crack model of the beam.

is from inertia force and the force derived from moving mass acceleration [35]. All of these terms are neglected in the moving load analysis.

The boundary conditions of the simply supported beam are

$$
\begin{gathered}
w(0, t)=w(L, t)=0, \\
w^{\prime \prime}(0, t)=w^{\prime \prime}(L, t)=0,
\end{gathered}
$$

and the initial conditions are

$$
w(x, 0)=\frac{\partial w(x, 0)}{\partial t}=0 .
$$

In the transverse cracked beam, it is divided into two segments, shown in Figure 2. The left segment of the crack has been treated as a beam connected to right by an elastic spring at the cracked section. The stiffness of the springs depends on the crack depth and geometry of the cracked section. The model used to present the crack leads to discontinuities in both vertical displacement and rotation due to bending, proportional to shear force and bending moment transmitted by the cracked section, respectively. The compatibility requirements enforce continuities of the displacement, bending moment, and shear force, respectively, across the crack and can be expressed as

$$
\begin{gathered}
w_{1}\left(x_{1}^{-}, t\right)=w_{2}\left(x_{1}^{+}, t\right), \\
E I w_{1}^{\prime \prime}\left(x_{1}^{-}, t\right)=E I w_{2}^{\prime \prime}\left(x_{1}^{+}, t\right), \\
w_{1}^{\prime \prime \prime}\left(x_{1}^{-}, t\right)=w_{2}^{\prime \prime \prime}\left(x_{1}^{+}, t\right),
\end{gathered}
$$

where $w_{1}$ and $w_{2}$ are the vertical displacement of left and right segment, respectively, and $x_{1}^{-}, x_{1}^{+}$denote the locations immediately after and before the crack position, respectively. The discontinuity condition at the crack for a simply connected beam can be written as [36]

$$
w_{2}^{\prime}\left(x_{1}^{+}, t\right)-w_{1}^{\prime}\left(x_{1}^{-}, t\right)=c_{\theta} h w_{2}^{\prime \prime}\left(x_{1}^{+}, t\right)
$$

where $h$ is the height of the beam and $c_{\theta}$ is functions depending on the crack ratio $\gamma(\gamma=a / h$, where $a$ is the crack depth) and the beam cross-section geometry. In the case of a rectangular section, this function can be written as [35, 37]

$$
\begin{aligned}
c_{\theta}= & 2\left(\frac{\gamma}{1-\gamma}\right)^{2} \\
& \cdot\left(5.93-19.69 \gamma+37.14 \gamma^{2}-35.84 \gamma^{3}+13.12 \gamma^{4}\right) .
\end{aligned}
$$

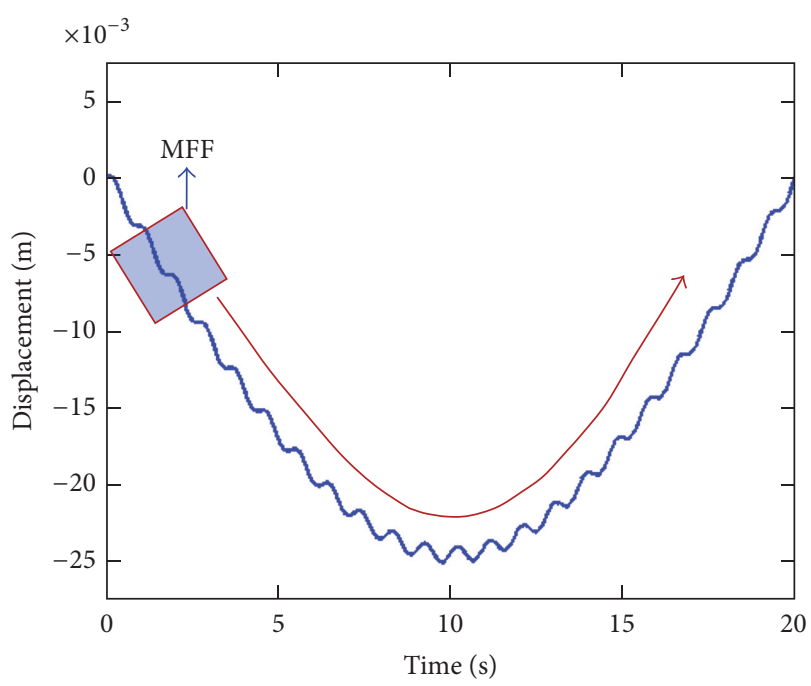

FIgURE 3: Schematic of Moving Filter Function (MFF).

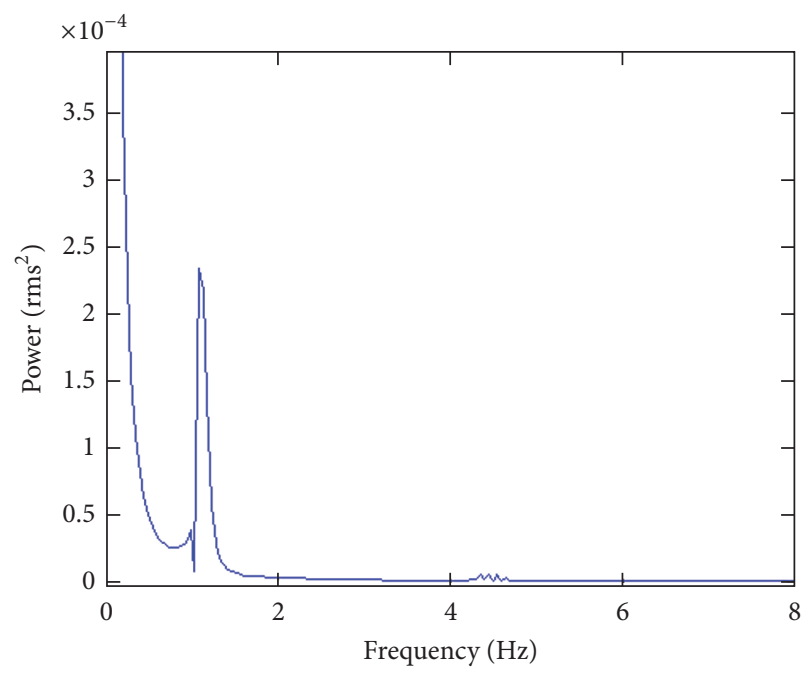

FIGURE 4: FFT spectrum of the response of undamaged beam with mass velocity for $1 \mathrm{~m} / \mathrm{s}$.

In the following simulation, using the method mentioned above, the response of different position of undamaged beam can be obtained with (10), while the transverse cracked beam is treated as two continuous beams with the connection condition as (14a), (14b), (14c), and (15), and the response of different position can be calculated as well. This responses are used in the following damage identification.

\section{Phase Space Reconstruction and the Damage Index}

3.1. The Phase Space Reconstruction of the Time Series. It is proved that any dynamic system can be completely recovered in a phase space, which may be reconstructed from the measured time domain response of the system [38]. The approach, 


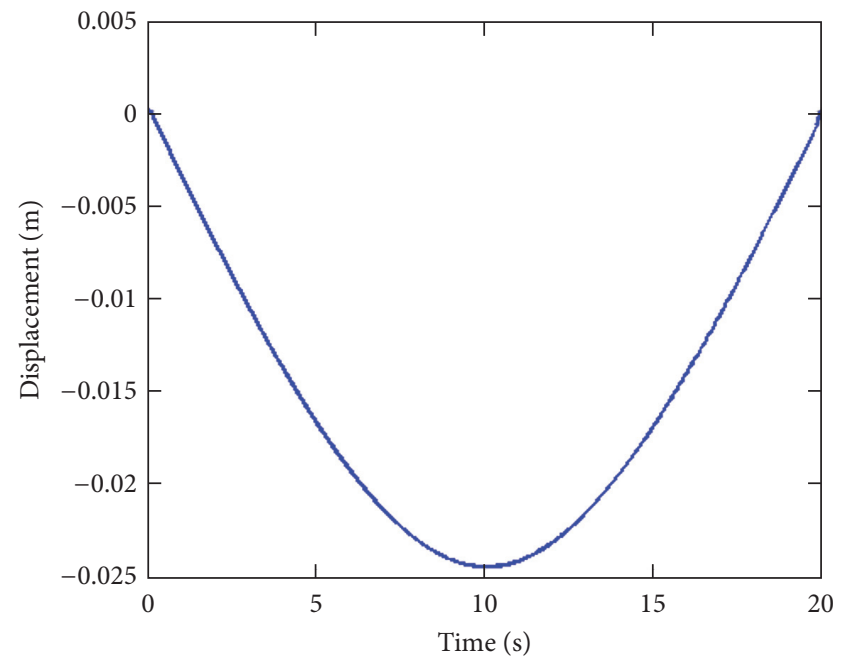

(a)

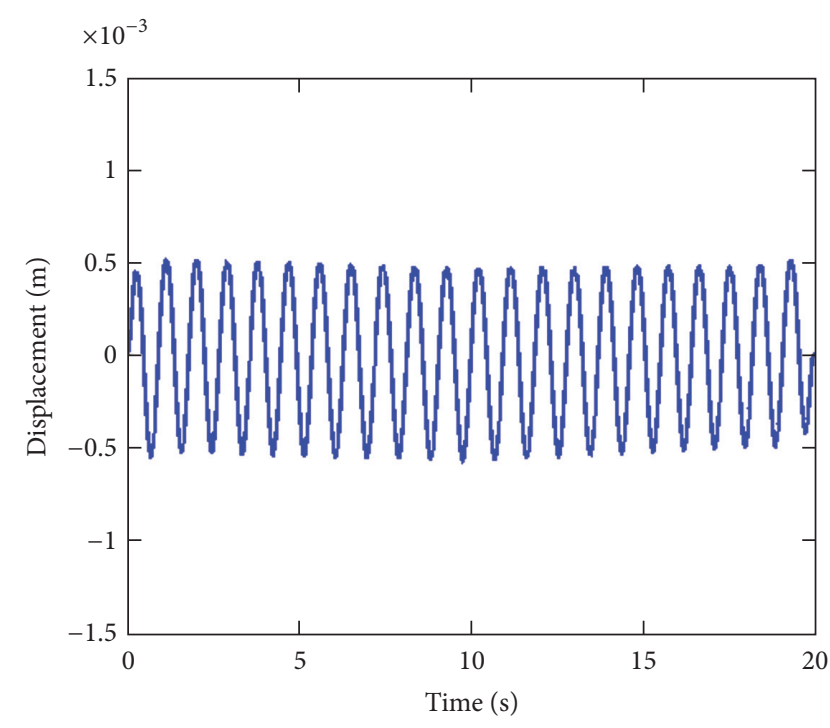

(b)

FIGURE 5: Displacement components of the undamaged beam with mass velocity for $1 \mathrm{~m} / \mathrm{s}$ : (a) the maintained component after using MFF twice and (b) the component with frequency for $1.07 \mathrm{~Hz}$.

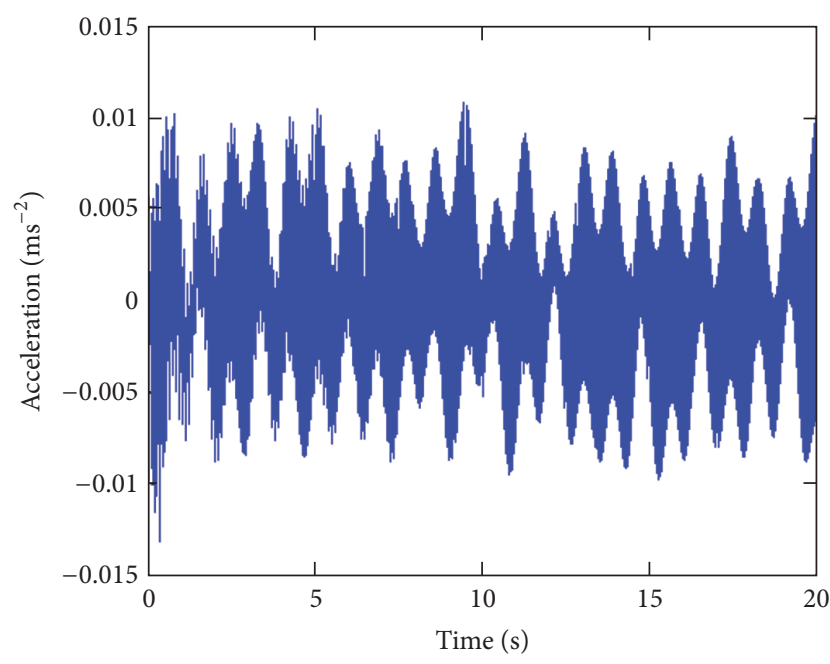

FIgURE 6: The midspan acceleration response of undamaged beam with mass velocity for $1 \mathrm{~m} / \mathrm{s}$.

commonly referred to as delay embedding, involves a concatenation of delayed copies of the measurement or set of measurements to represent additional state vectors up to a sufficient dimension, such that the dynamics of the system are adequately captured. Assume an $N$ length response data, $a(n), n=1, \ldots, N$, has been collected; the discrete time instance of the phase space is given by

$$
a(n)=[a(n), a(n+\mathrm{Tau}), \ldots, a(n+(z-1) \mathrm{Tau})],
$$

where the delayed copies of $a(n)$ are often referred to as the pseudostate vectors. A successful reconstruction requires the choice of both delay Tau and embedding dimension $z$. The autocorrelation function is usually used to determine the delay. The time of first zero or crossing of the autocorrelation function is used to select the delay. The normalized autocorrelation function is calculated by [39]

$$
c_{\mathrm{ss}}(\mathrm{Tau})=\frac{\sum_{n=0}^{N-1}[s(n)-\bar{s}][s(n+\mathrm{Tau})-\bar{s}]}{\sum_{n=0}^{N-1}[s(n)-\bar{s}]^{2}},
$$

where $\bar{s}$ is the mean value of the time series $s$. It is proved that the singular system analysis is a good approach to choose the embedding dimension $z$ [31]. The singular system analysis proceeds by first forming the matrix, given as (19), made up of the time series with $N$ time points.

$$
\mathbf{W}=\left[\begin{array}{cccc}
a(1) & a(2) & \cdots & a(q) \\
a(2) & a(3) & \cdots & a(q+1) \\
\vdots & \vdots & \ddots & \vdots \\
a(N-q) & a(N-q+1) & \cdots & a(N)
\end{array}\right],
$$

where $q$ defines the time window $\tau=q / f_{s}$ for sample frequency $f_{s}$. Choice of $\tau$ is dependent on the frequency content of the signal; one may choose this window as $\tau \leq$ $1 / f^{*}$, where $f^{*}$ is the band-limiting frequency above which the Fourier spectrum of the time series contains no significant power. The singular value decomposition of $\mathbf{W}$ is computed as

$$
\operatorname{SVD}(\mathbf{W})=\mathbf{U}_{N \times q} \mathbf{S}_{q \times q} \mathbf{V}_{q \times q}^{T}
$$

where the singular values are given by the diagonals of the matrix $\mathbf{S}$. The number of large singular values (not close to zero) is selected to be the embedding dimension $z$. The details of the method of choosing appropriate Tau and $z$ are described in $[1,40]$. 


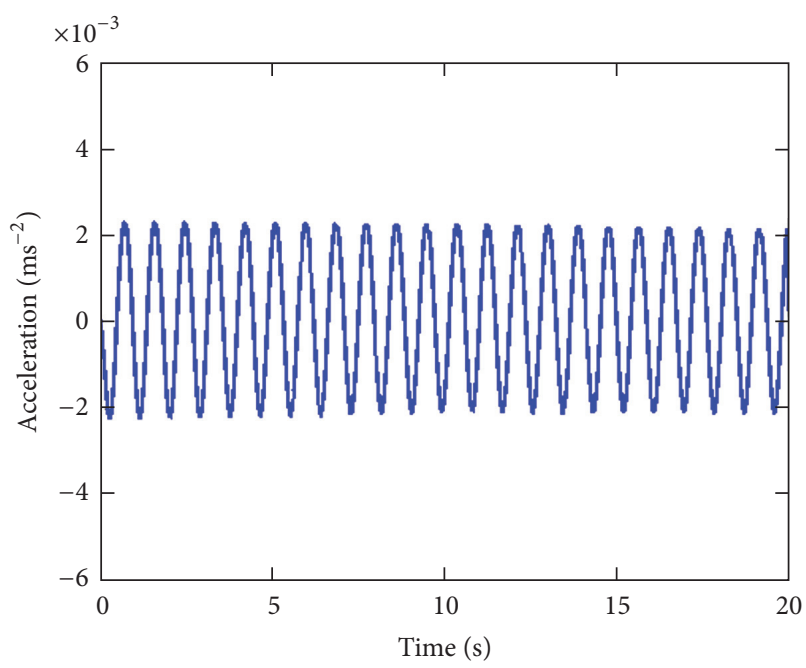

(a)

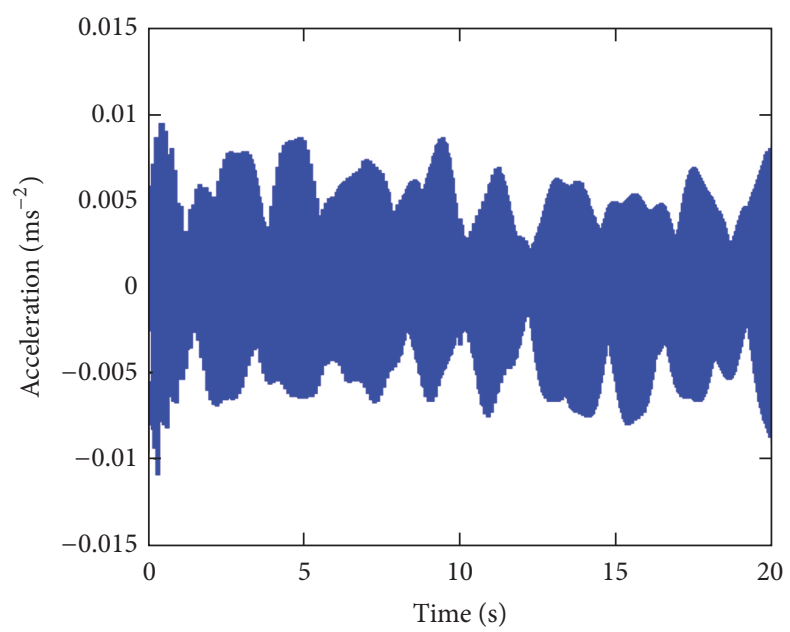

(b)

FIGURE 7: Acceleration components of the undamaged beam with mass velocity for $1 \mathrm{~m} / \mathrm{s}$ : (a) the maintained component with frequency for $1.07 \mathrm{~Hz}$ and (b) the high dynamic component with frequency for $4.44 \mathrm{~Hz}$.

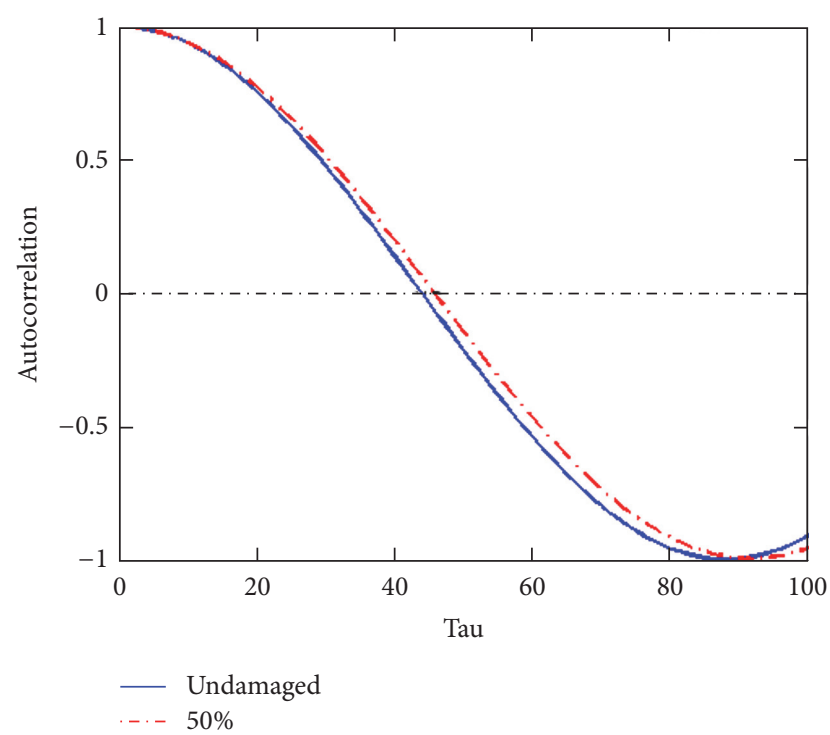

FIGURE 8: Autocorrelation function curve of the scenario with mass is $200 \mathrm{Kg}$ and velocity is $1 \mathrm{~m} / \mathrm{s}$ : blue curve is for undamaged condition and red curve is for the $50 \%$ damaged one.

3.2. Phase Space-Based Damage Feature Extraction. The damage feature is based on the previous study [1] but some improvements are made. The phase trajectory corresponding to the signals from damaged structure as defined by (17) can be described as

$$
\mathbf{d}(n)=[d(n), d(n+\mathrm{Tau}), \ldots, d(n+(z-1) \mathrm{Tau})] .
$$

The damage feature extraction is to construct the metric capable of discriminating between the undamaged trajectory a and damaged trajectory $\mathbf{d}$. If the time series is embedded

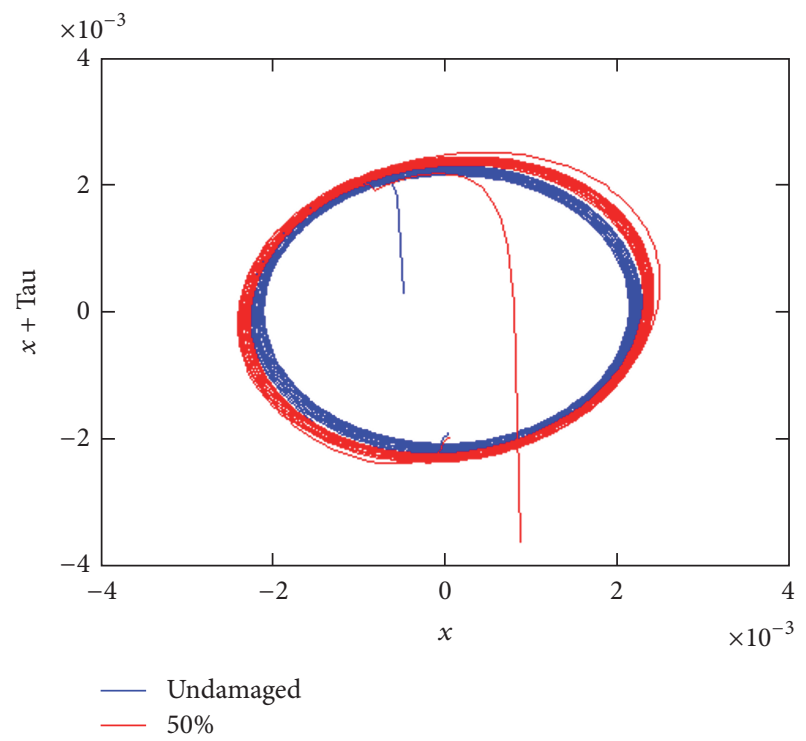

FIGURE 9: Phase spaces of the scenario with mass is $200 \mathrm{Kg}$ and velocity is $1 \mathrm{~m} / \mathrm{s}$ : blue curve is for undamaged condition and red curve is for the $50 \%$ damaged one.

with $z$ dimensions, the coordinators of undamaged phase space can be described as

$$
\begin{gathered}
A(n)=[a(1), a(2), \ldots, a(N-(z-1) \mathrm{Tau})] \\
A(n+\mathrm{Tau})=[a(1+\mathrm{Tau}), a(2+\mathrm{Tau}), \ldots, \\
a(N-(z-2) \mathrm{Tau})] \\
\vdots \\
A(n+(z-1) \mathrm{Tau})=[a(1+(z-1) \mathrm{Tau}), \\
\quad a(2+(z-1) \mathrm{Tau}), \ldots, a(N)] .
\end{gathered}
$$




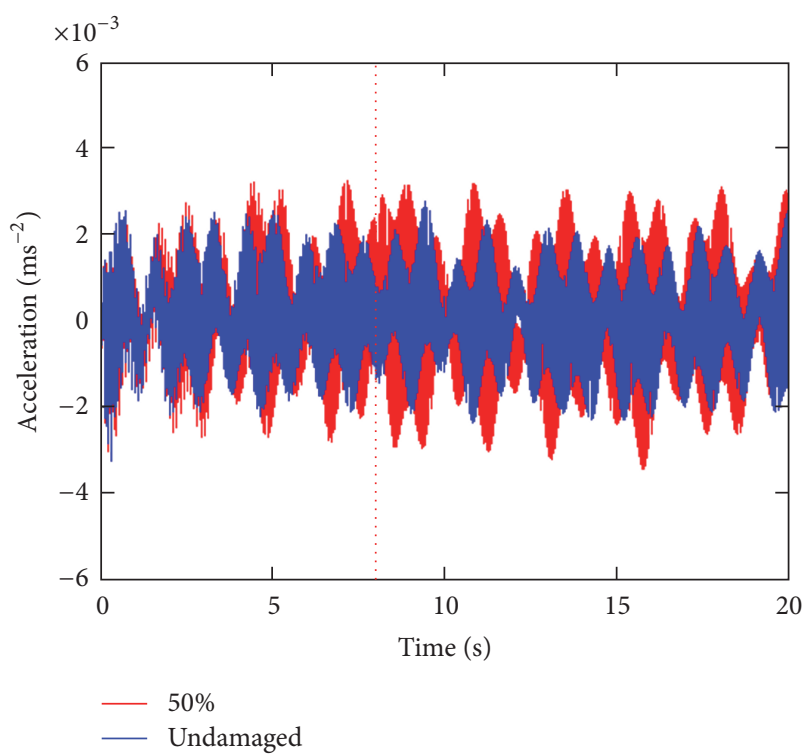

(a)

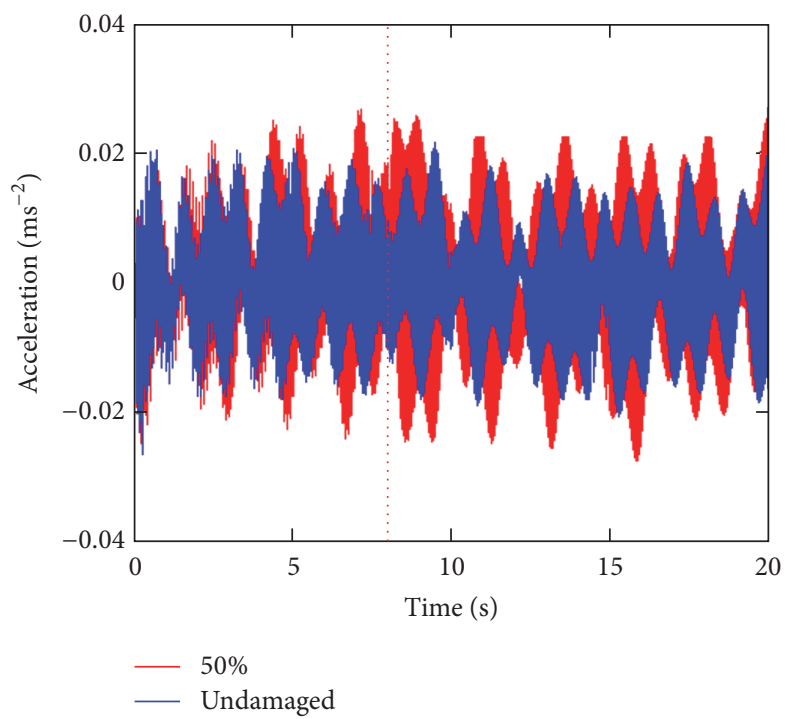

(b)

Figure 10: Acceleration responses: (a) the mass is $50 \mathrm{Kg}$ and (b) the mass is $400 \mathrm{Kg}$.

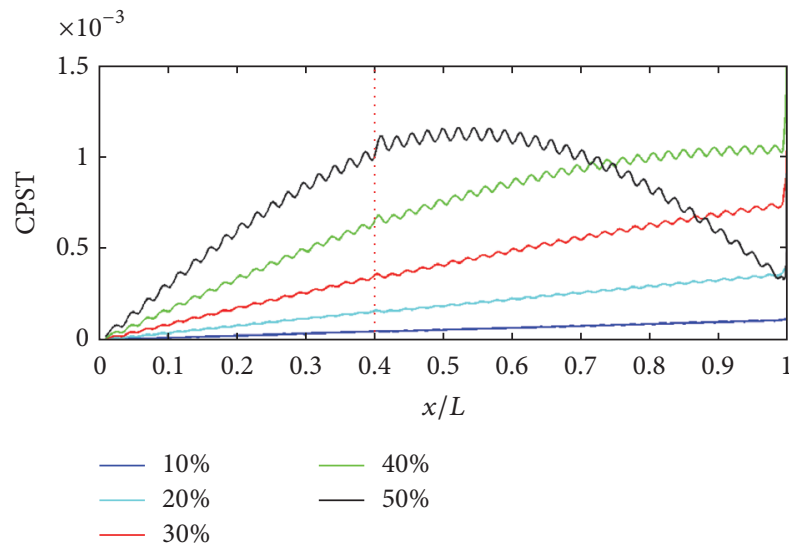

FIGURE 11: Unfiltered CPST of different damage levels using acceleration responses with the mass which is $50 \mathrm{Kg}$.

The damaged dataset $\mathbf{d}$ can be also reconstructed using (22). The points on the trajectory corresponding to the damaged and undamaged structure are selected with time index $r$ as $D(r)$ and $A(r)$. The damage index CPST (change of phase space topology) of this time is defined as

$$
\operatorname{CPST}(r)=\|A(r)-D(r)\|, \quad r=1, \ldots, N-z \cdot \mathrm{Tau},
$$

where $\|\cdot\|$ operator computes the Euclidean norm of point $D(r)$ and $A(r)$. In this study, the embedding dimension is selected to be 2. Trickey et al. [41] have demonstrated that exciting a structure with the output of a narrow-band stochastic process can result in a structural response which is practically low-dimensional and easily modeled in phase space.

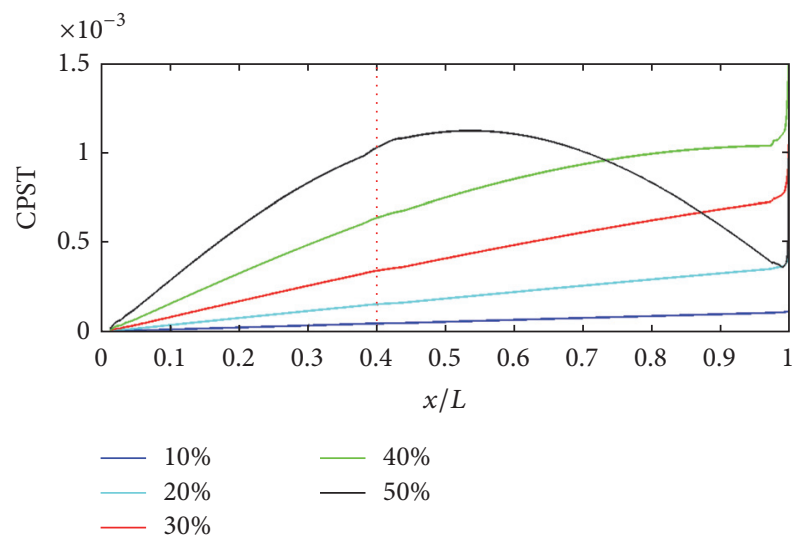

Figure 12: Filtered CPST of different damage levels using acceleration responses with the mass which is $50 \mathrm{Kg}$.

\section{Damage Detection for Beam-Like Structure under a Moving Mass}

4.1. Numerical Model. To further demonstrate the proposed method in identifying the structural damage, a simple supported beam as shown in Figure 1 is employed as an example. The aim is to evaluate the sensitivity of the method to the crack depth ratio $\gamma$, crack location $x_{1}$, sensor location $L_{s}\left(L_{s}\right.$ is the relative position from the left to right end of the beam), mass of the moving mass $m_{m}$, the velocity of the moving mass $v$, and multiple cracks. In addition, the robustness to the ambient noise is also evaluated. It should be noted that all the study in this paper is based on the simulation, completely neglecting the effect of instrumental errors. 


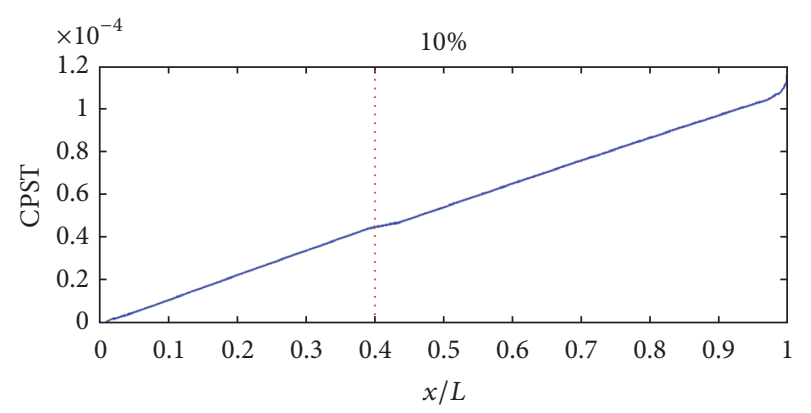

FigURE 13: CPST of $10 \%$ damaged scenario using acceleration response with the mass which is $50 \mathrm{Kg}$.

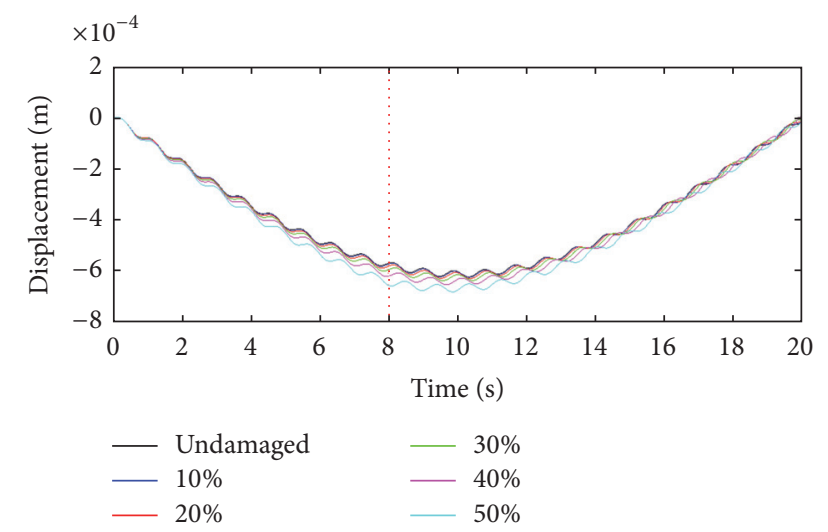

FIGURE 14: Displacement responses of different damage levels with the mass which is $50 \mathrm{Kg}$.

The physical-geometric parameters of example beam model are as follows: length of beam is $L=20 \mathrm{~m}$, height is $h=0.2 \mathrm{~m}$, width is $b=0.1 \mathrm{~m}$, Young's modulus is $E=$ $200 \mathrm{GPa}$, and material density is $\rho=7850 \mathrm{Kgm}^{-3}$. The moving mass is used to simulate a passing vehicle with different velocity. A crack with the ratio $\gamma$ equal to $10 \%, 20 \%, 30 \%$, $40 \%$, and $50 \%$ at the position of $L_{d}\left(L_{d}=x_{1} / L\right.$ is the relative length) equal to $0.2,0.4,0.6$, and 0.8 length from the left support, respectively, as shown in Figure 1, is assumed in the beam in numerical simulations. The displacement and acceleration responses at the same measurement point are calculated and used in the analysis for damage identification.

In this paper, six analysis scenarios are analyzed. Scenario 1 is that the mass of the moving mass varies, crack location, sensor location, and mass velocity is maintained at a constant value, so as to analyze the effectiveness of the proposed method to the mass magnitude. In scenario 2, the crack location is set to be different; others factors are maintained at a constant value to analyze the effect of different damage location conditions. Scenario 3 is to demonstrate the effectiveness of the proposed method using the response measured with a single sensor installed at different location of the beam. Here it should be noted that, in the previous studies [12-19], authors only used the response of midspan of the beam in damage identification but did not try to use the responses of others positions. Scenario 4 is to evaluate the effectiveness of mass velocities, and scenario 5 is to evaluate the effectiveness

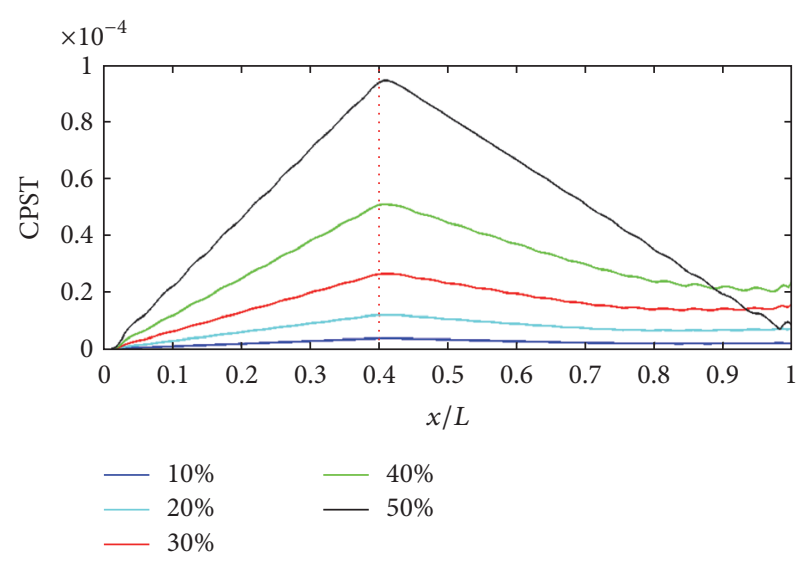

FIgURE 15: CPST of 10\% damaged scenario using displacement responses with the mass which is $50 \mathrm{Kg}$.

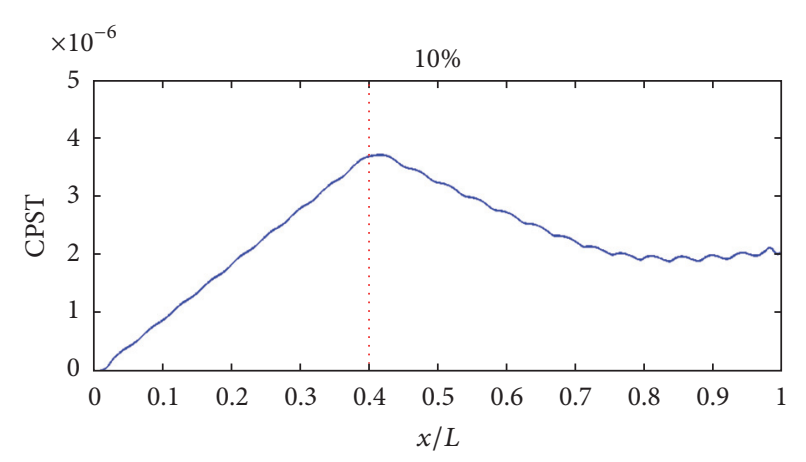

FIGURE 16: CPST of $10 \%$ damaged scenario using displacement response with the mass which is $50 \mathrm{Kg}$, and the dotted line is for the damage location.

of the proposed method for the multicracked beam. Scenario 6 is to demonstrate the robustness of the index to noise by smearing the Gauss noise with different level in the responses. The details of the five scenarios are shown in Table 1.

The natural frequencies of all the damage scenarios are also calculated (when $p(x, t)$ is equal to zero in (1)), shown in Table 2. It should be note that the frequencies of scenarios with the damage location $L_{d}$ equal to 0.6 and 0.8 are the same as the scenarios with $L_{d}$ equal to 0.4 and 0.2 , respectively. Hence, Table 2 only shows the results of the scenarios with $L_{d}$ is 0.2 and 0.4. As shown, between the two damage location scenarios, the natural difference of the frequencies is small compared with the same damage levels.

4.2. Moving Filter Function. Before phase space reconstructing, the responses should be treated to remove the high dynamic information. A Moving Filter Function (MFF) is defined to provide a robust approach to remove the high dynamic component and crucially has the advantage of maintaining the low dynamic information. An MFF replaces each point in the signal, $a(i)$, at an instant $i$, with the average $\bar{a}(i)$ of several adjacent points. More precisely the average is 


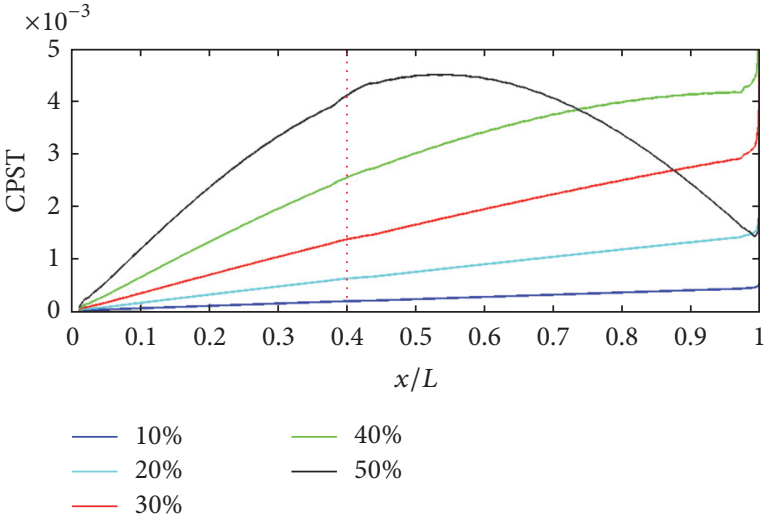

(a)

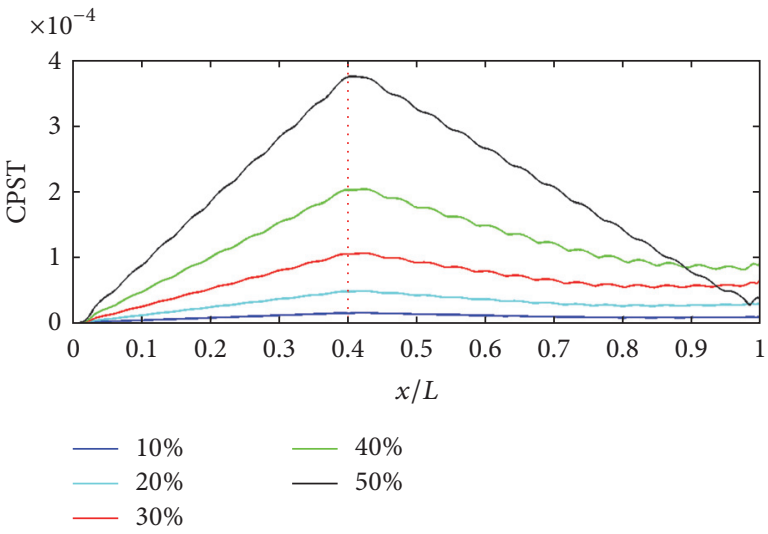

(b)

FIGURE 17: CPST of different damage levels with the mass which is $200 \mathrm{Kg}$ : (a) is for the acceleration responses and (b) is for the displacement responses.

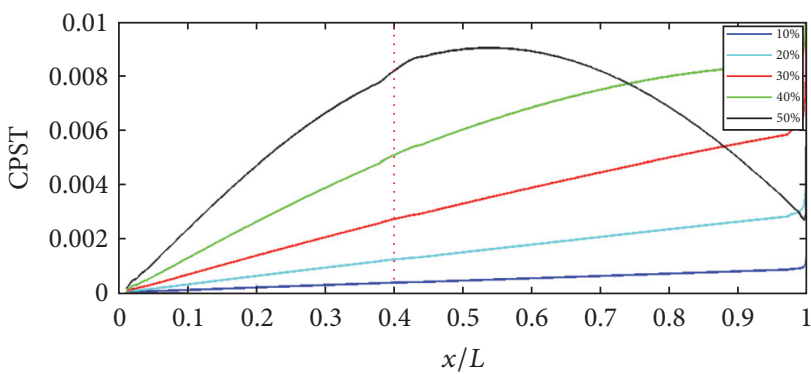

(a)

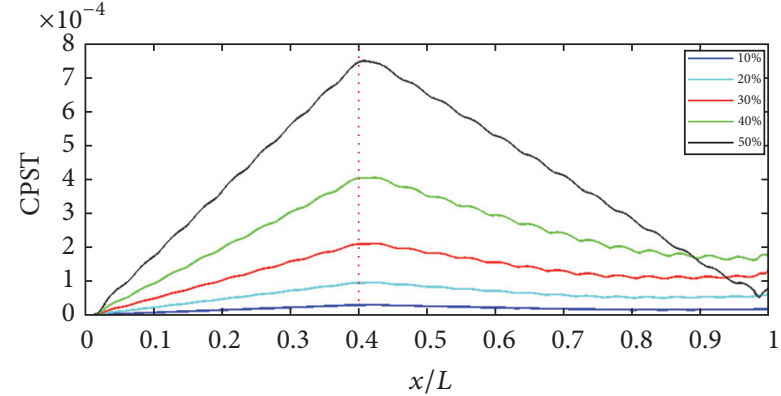

(b)

FIGURE 18: CPST of different damage levels with the mass which is $400 \mathrm{Kg}$ : (a) is for the acceleration responses and (b) is for the displacement responses.

TABLE 1: Analysis scenarios.

Factor to be used to

evaluate the sensitivity

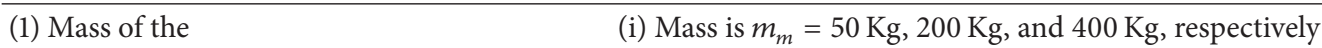
moving mass

\section{(2) Crack location}

(3) Sensor location

(4) Moving mass velocity

(5) Multiple damage

(6) Robustness to noise
Condition of the considered factors

(ii) Crack location is $L_{d}=0.4$ (iii) Sensor location is $L_{s}=0.5$ (iv) Velocity is $1 \mathrm{~m} / \mathrm{s}$

(i) Crack location is $L_{d}=0.2,0.4,0.6$, and 0.8 , respectively

(ii) Mass is $m_{m}=200 \mathrm{Kg}$

(iii) Sensor location is $L_{s}=0.5$ (iv) Velocity is $1 \mathrm{~m} / \mathrm{s}$

(i) Sensor location is $L_{s}=0.25,0.5$, and 0.75 , respectively

(ii) Mass is $m_{m}=200 \mathrm{Kg}$

(iii) Crack location is $L_{d}=0.4$ (iv) Velocity is $1 \mathrm{~m} / \mathrm{s}$

(i) The moving mass velocity is $0.5 \mathrm{~m} / \mathrm{s}, 1 \mathrm{~m} / \mathrm{s}, 5 \mathrm{~m} / \mathrm{s}$, and $10 \mathrm{~m} / \mathrm{s}$, respectively

(ii) Crack location is $L_{d}=0.4$ (iii) Sensor location is $L_{s}=0.5$

(iv) Mass is $m_{m}=200 \mathrm{Kg}$

(i) The moving mass velocity is $1 \mathrm{~m} / \mathrm{s}$

(ii) Crack locations are $L_{d}=0.3(\gamma=20 \%)$ and $0.6(\gamma=10 \%)$, respectively

(iii) Sensor location is $L_{s}=0.5$ (iv) Mass is $m m=200 \mathrm{Kg}$

(i) The noise level is $5 \%, 10 \%$, and $30 \%$ respectively

(ii) Mass is $m_{m}=200 \mathrm{Kg}$

(iii) Crack location is $L_{d}=0.4$ (iv) Sensor location is $L_{s}=0.5$

(v) Mass velocity is $1 \mathrm{~m} / \mathrm{s}$ 


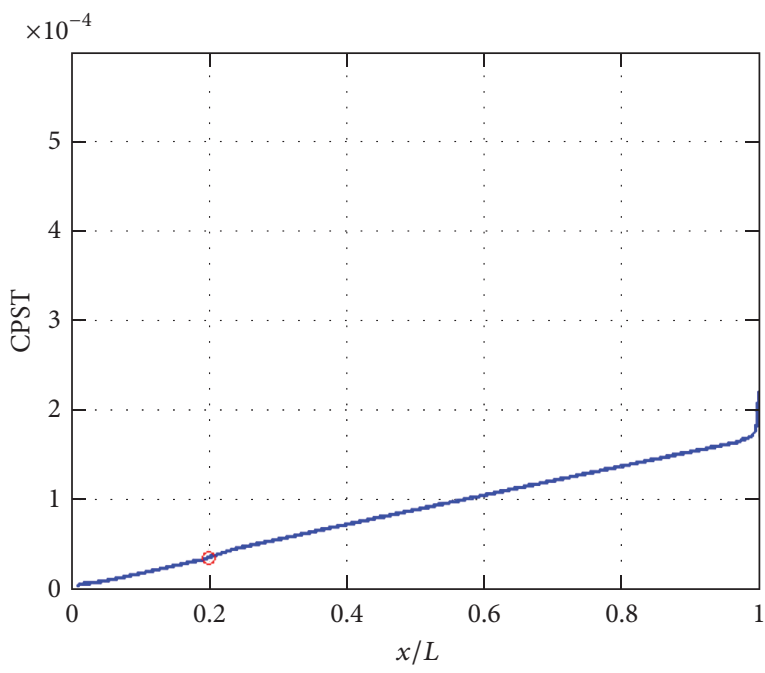

(a)

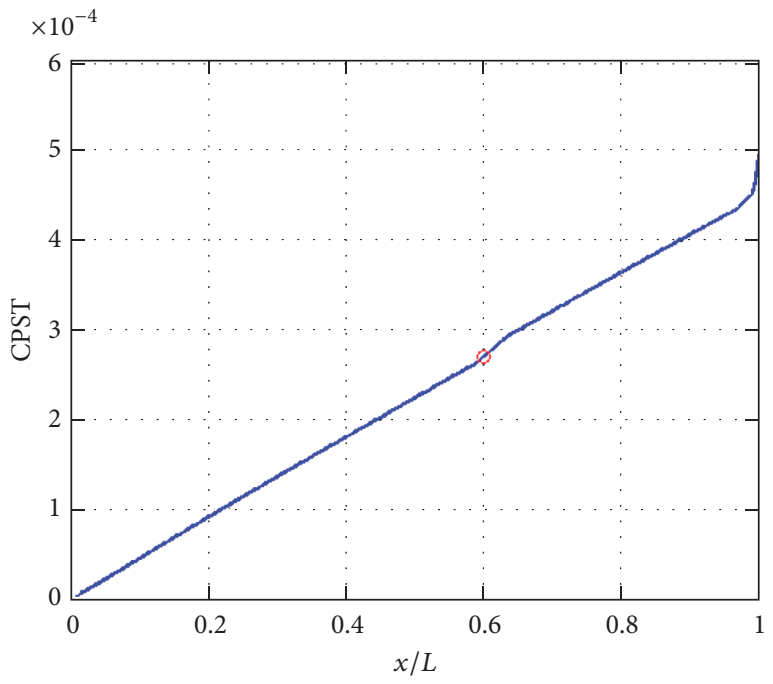

(c)

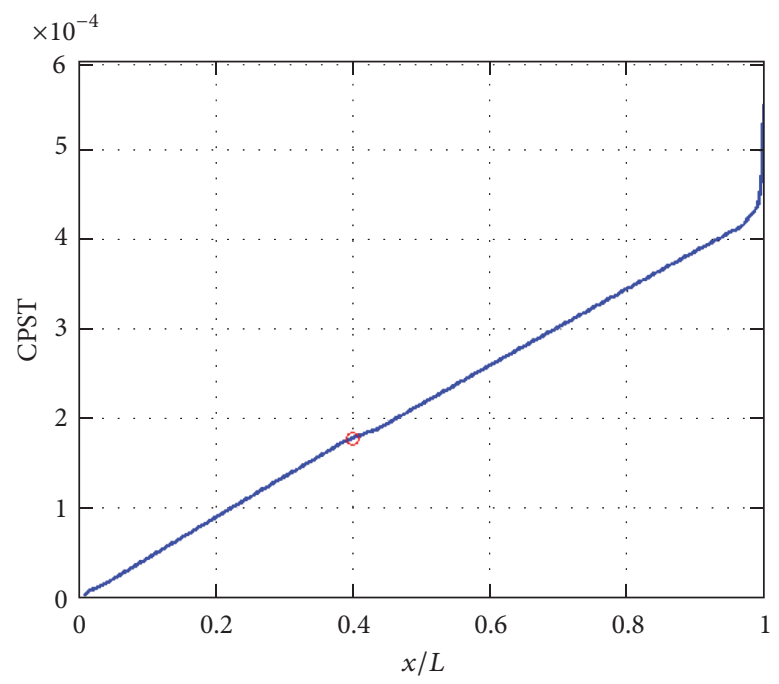

(b)

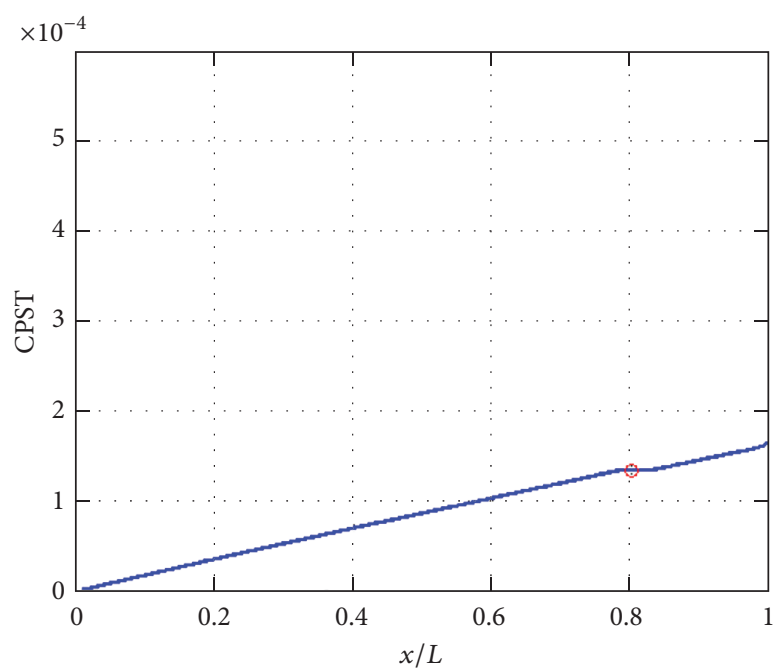

(d)

FIGURE 19: CPST of 10\% damaged scenario using acceleration responses with the mass which is $200 \mathrm{Kg}$ and velocity which is $1 \mathrm{~m} / \mathrm{s}$ : (a) damage location is $L_{d}=0.2$, (b) damage location is $L_{d}=0.4$, (c) damage location is $L_{d}=0.6$, and (d) damage location is $L_{d}=0.8$.

TABLE 2: Natural frequencies $(\mathrm{Hz})$ of different damage scenarios.

\begin{tabular}{lcccccccccccc}
\hline \multirow{2}{*}{ Mode number } & \multicolumn{9}{c}{$L_{d}=0.2$} & \multicolumn{4}{c}{$L_{d}=0.4$} & \multicolumn{4}{c}{$c$} \\
& $0 \%$ & $10 \%$ & $20 \%$ & $30 \%$ & $40 \%$ & $50 \%$ & $0 \%$ & $10 \%$ & $20 \%$ & $30 \%$ & $40 \%$ & $50 \%$ \\
\hline 1 & 1.14 & 1.14 & 1.14 & 1.14 & 1.13 & 1.13 & 1.14 & 1.14 & 1.14 & 1.13 & 1.12 & 1.10 \\
2 & 4.57 & 4.56 & 4.55 & 4.52 & 4.47 & 4.39 & 4.57 & 4.57 & 4.56 & 4.55 & 4.53 & 4.50 \\
3 & 10.26 & 10.24 & 10.21 & 10.15 & 10.05 & 9.89 & 10.26 & 10.26 & 10.24 & 10.22 & 10.18 & 10.12 \\
4 & 18.18 & 18.17 & 18.14 & 18.11 & 18.04 & 17.93 & 18.18 & 18.16 & 18.10 & 18.00 & 17.84 & 17.58 \\
5 & 28.27 & 28.27 & 28.27 & 28.27 & 28.27 & 28.27 & 28.27 & 28.27 & 28.27 & 28.27 & 28.27 & 28.27 \\
\hline
\end{tabular}

calculated here from an equal number of data points on either side of a central value as

$$
\bar{a}(i)=\frac{1}{k} \sum_{j=i-k / 2}^{j=i+k / 2} a(i+j),
$$

where $k$ defines the span of the moving function and it can be calculated by

$$
k=T_{k} f_{s}
$$




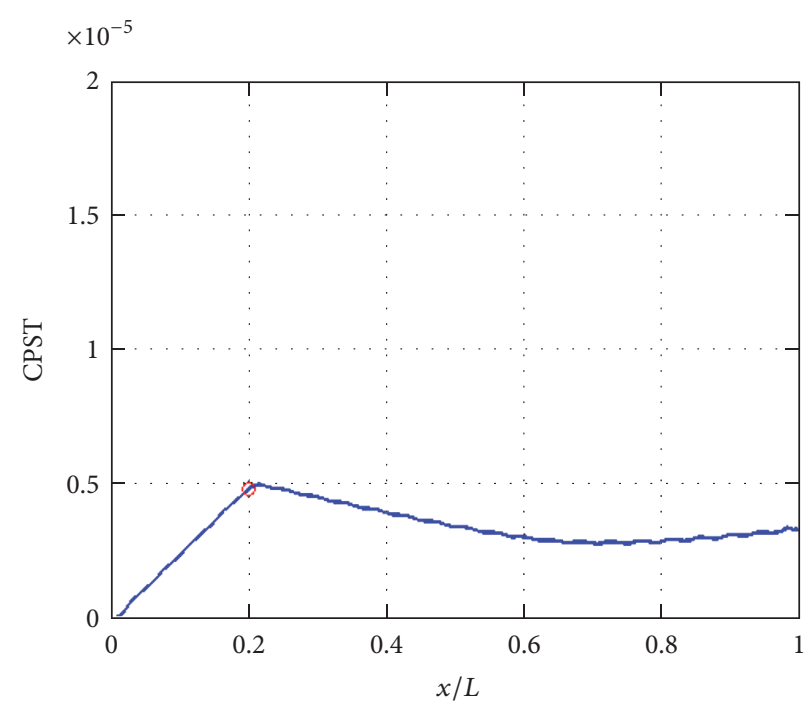

(a)

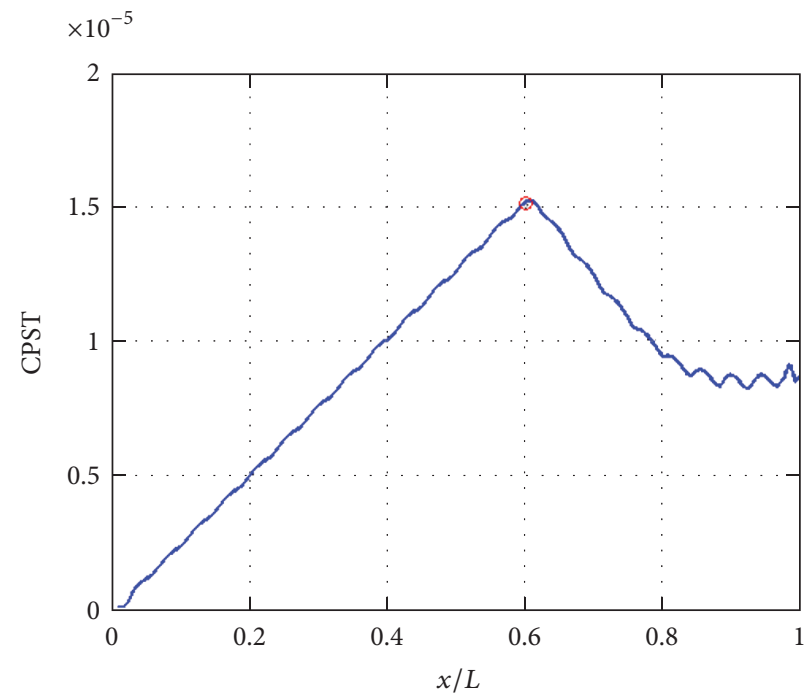

(c)

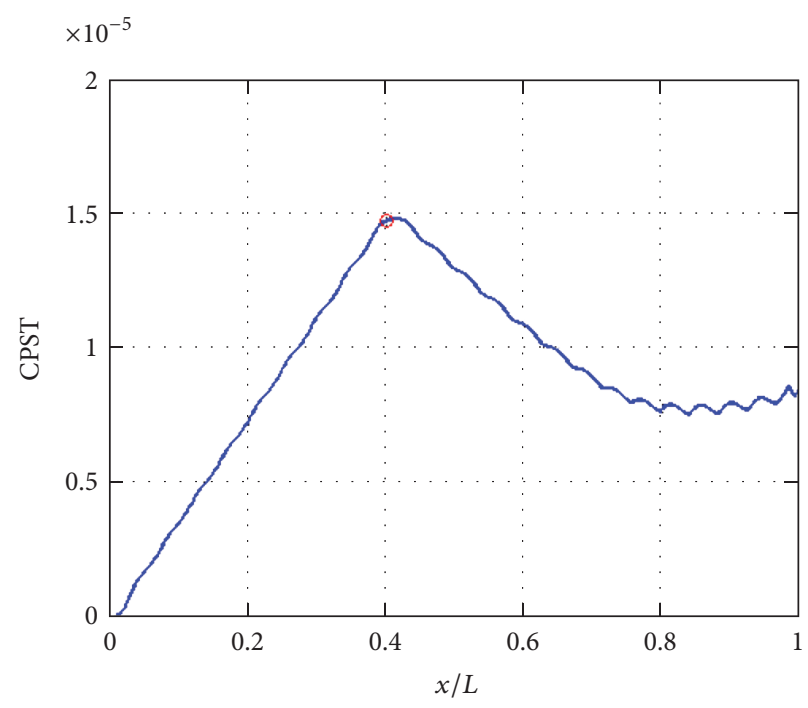

(b)

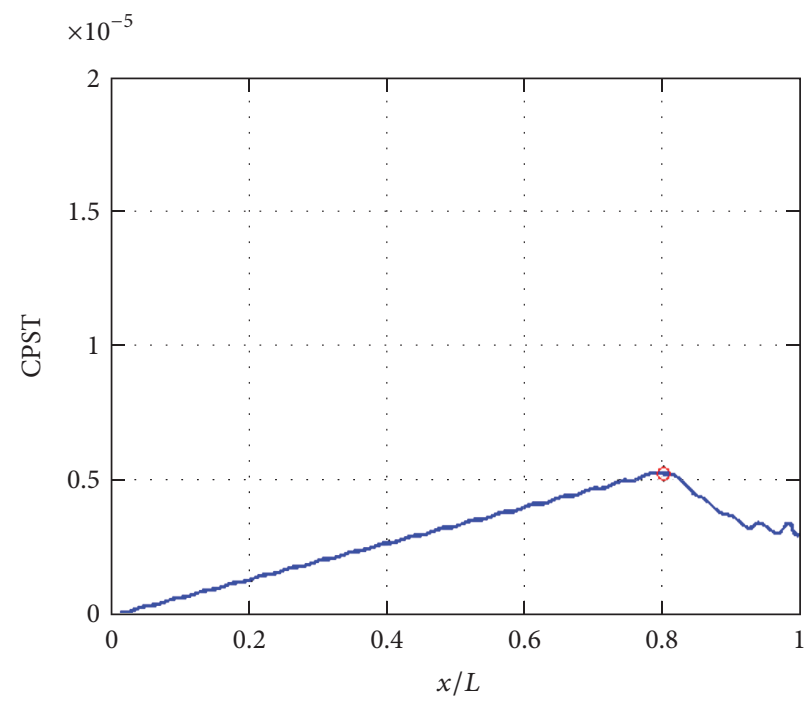

(d)

FIGURE 20: CPST of $10 \%$ damaged scenario using displacement responses with the mass which is $200 \mathrm{Kg}$ and velocity which is $1 \mathrm{~m} / \mathrm{s}$ : (a) damage location is $L_{d}=0.2$, (b) damage location is $L_{d}=0.4$, (c) damage location is $L_{d}=0.6$, and (d) damage location is $L_{d}=0.8$.

where $T_{k}$ is the period of the dynamic information to be removed and $f_{s}$ is the sampling frequency. Then the responses are scanned by MFF along the length direction with the different $T_{k}$ from large to small, respectively. Hence, the responses are analyzed by the Fast Fourier Transform (FFT) algorithm to find the dominant dynamic component and the high dynamic information with low power; the corresponding frequencies $\left(1 / T_{k}\right)$ are obtained as well. This method can be also used to separate the signal. A schematic view of the MFF is shown in Figure 3.

For example, when moving mass is $200 \mathrm{Kg}$ and velocity is $1 \mathrm{~m} / \mathrm{s}$, the midspan displacement response of undamaged beam is shown as the blue curve in Figure 3. The dominant frequency of vibration of this response shown in Figure 4 is found to be $1.07 \mathrm{~Hz}$, while another higher dynamic frequency of this signal is $4.44 \mathrm{~Hz}$. Comparing to the natural frequencies as shown in the Table 2, the frequencies extracted from the signal of undamaged beam are close to the mode 1 and mode 2 frequencies, but relatively less than the natural frequencies, respectively. This is because the frequencies are the central frequencies of the signals of the beam subjected to a moving mass. The vibration frequencies are effected by the interaction between moving mass and beam.

As shown in Figure 4, the power of dynamic component with $4.44 \mathrm{~Hz}$ is much less than that of the dominant dynamic information. This component is erased while the dominant ones to be used in the damage detection are retained. The dynamic period of vibration of higher dynamic component is $0.225 \mathrm{~s}(1 / 4.44 \mathrm{~s})$. The sampling frequency used to generate the displacement responses is $2000 \mathrm{~Hz}$; consequently an MFF with a $k$ value of $450(2000 * 0.225)$ points will remove this component. 


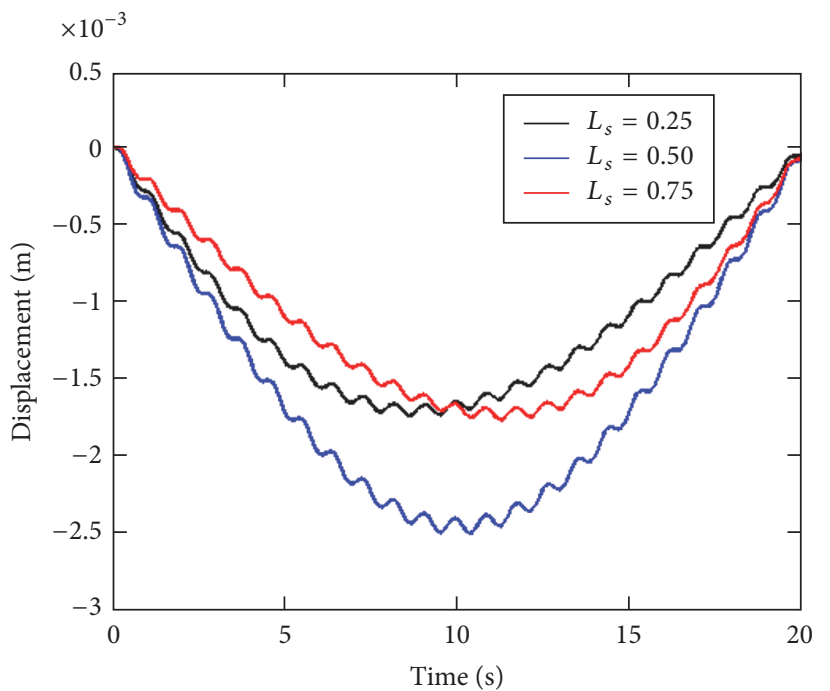

(a)

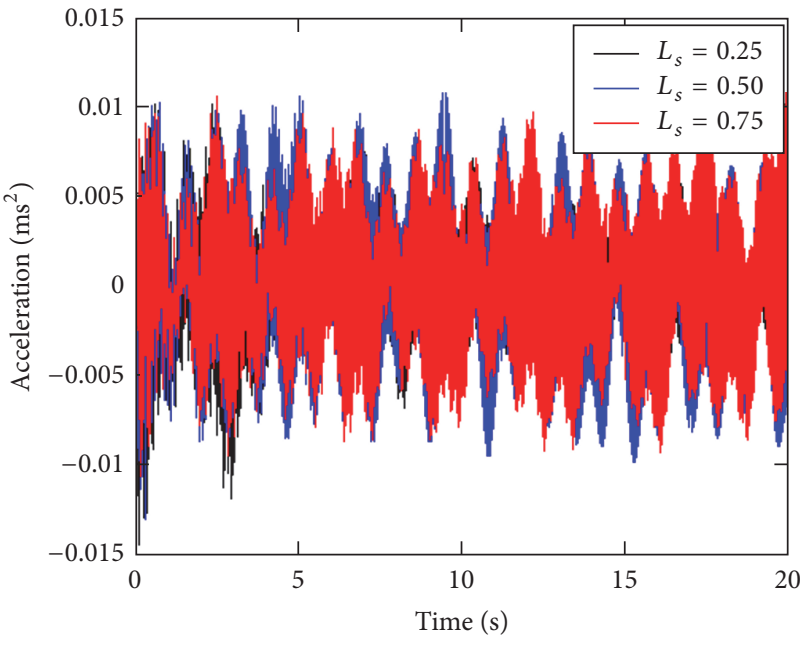

(b)

FIGURE 21: Undamaged responses of different sensor location scenarios with $v=1 \mathrm{~m} / \mathrm{s}, m_{m}=200 \mathrm{Kg}$, and $L_{d}=0.4$ : (a) is displacement responses and (b) is acceleration responses.

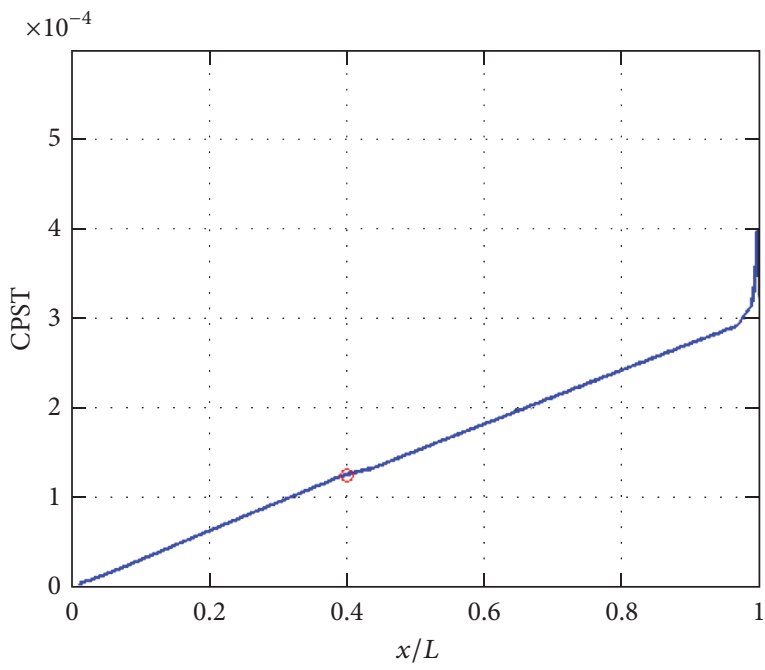

(a)

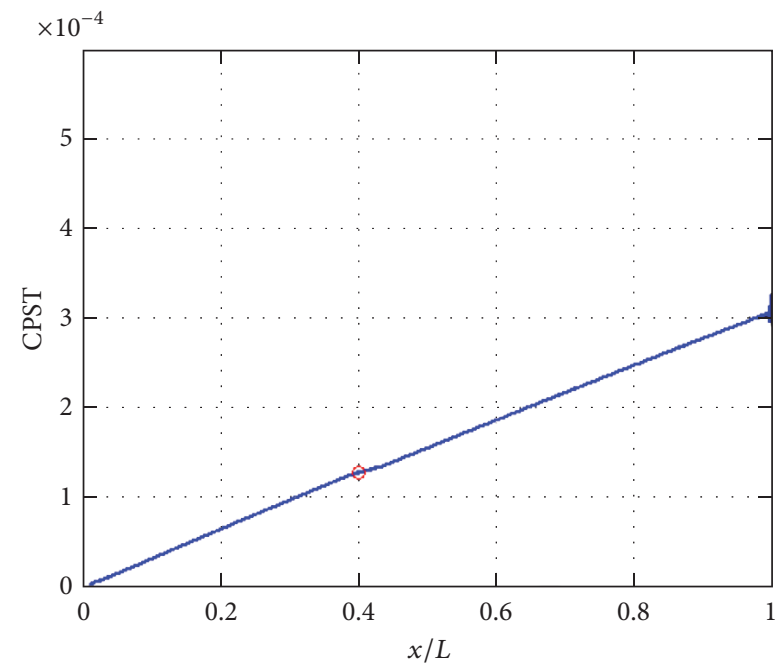

(b)

FIGURE 22: CPST of different sensor location scenarios using acceleration responses with damaged level is $10 \%$, mass is $200 \mathrm{Kg}$, and velocity is $1 \mathrm{~m} / \mathrm{s}$ : (a) sensor location is $L_{s}=0.25$ and (b) sensor location is $L_{s}=0.75$.

In order to separate the dominant dynamic component with the central frequency of $1.07 \mathrm{~Hz}$, the MFF is used again; corresponding to the span, $k$ is $1869((1 / 1.07 \mathrm{~Hz}) * 2000)$ points. The final maintained result of undamaged response is shown in Figure 5(a), and the dominant dynamic component is shown in Figure 5(b). Result shown in Figure 5(a) is the deflection of the midspan point caused by the mass gravity, while the result shown in Figure 5(b) is the dynamic component of interaction between the mass and beam.

The corresponding acceleration response of this example is shown in Figure 6. Using MFF, the signal is separated to two components shown in Figure 7. The component with central dynamic frequencies for $1.07 \mathrm{~Hz}$ is shown in Figure 7(a) and the higher dynamic component is shown in Figure 7(b). The maintained displacement and acceleration responses after removing the high dynamic components are used in the following damage detection, and the results of the two types of responses are compared.

\subsection{Damage Localization}

4.3.1. Phase Space Reconstruction. Using the phase space reconstruction method described in Section 3, the filtered responses are translated from time domain into the spatial domain. Here taking the midspan acceleration responses, for example, again. The maintained response to be used 


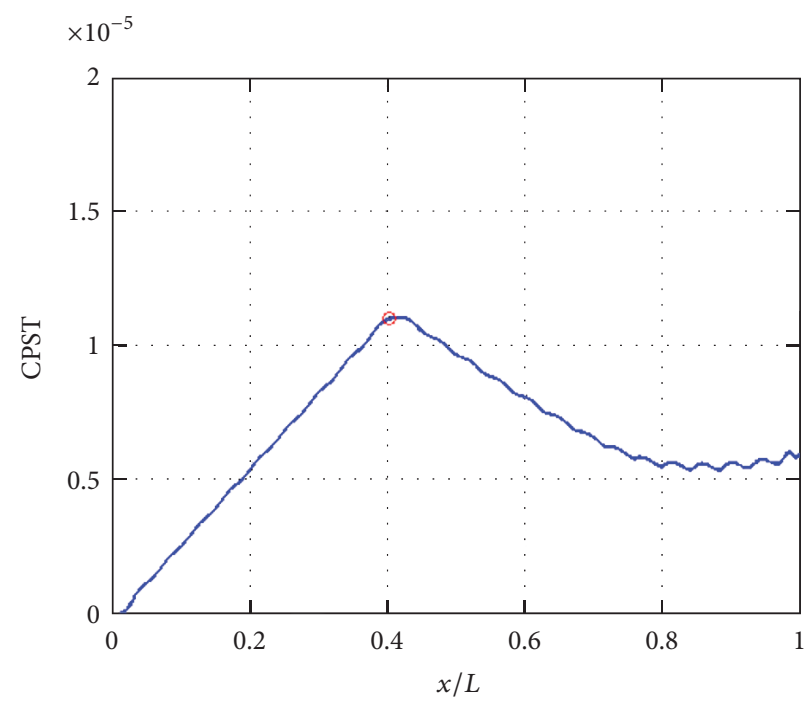

(a)

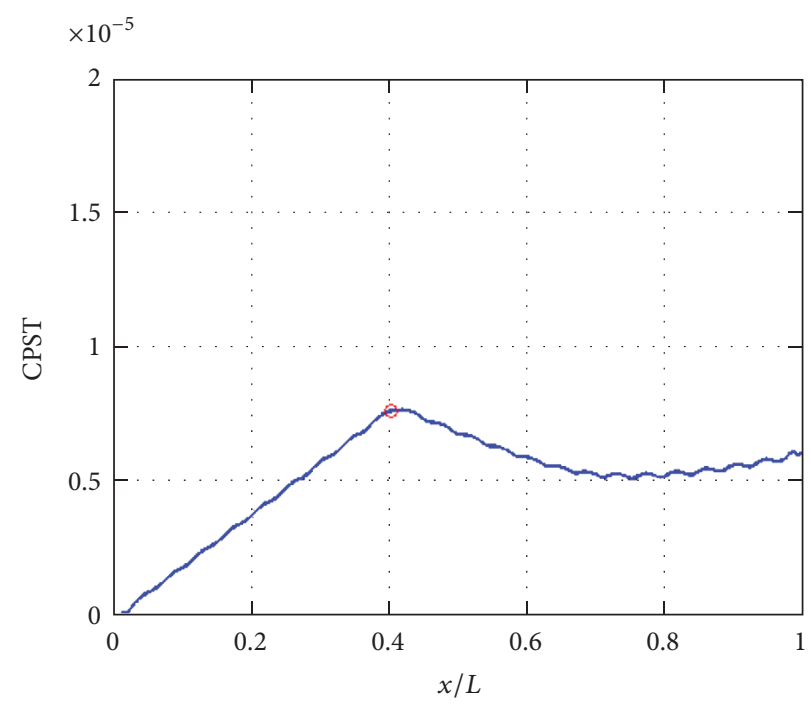

(b)

FiguRE 23: CPST of different sensor location scenarios using displacement responses with damaged level is $10 \%$, mass is $200 \mathrm{Kg}$, and velocity is $1 \mathrm{~m} / \mathrm{s}$ : (a) sensor location is $L_{s}=0.25$ and (b) sensor location is $L_{s}=0.75$.

in damage detection is that the high dynamic component is removed. Hence, the example in Figure 6 is the initial response, Figure $7(\mathrm{~b})$ is the component which removed the high dynamic components, and Figure $7(\mathrm{a})$ is the maintained response. The autocorrelation function curves of the maintained component are shown in Figure 8. The blue curve is for the undamaged one and the red dotted line is for the $50 \%$ damaged one. As shown, 50\% damaged curve deviates from undamaged one and the first points crossing zero of the autocorrelation function of undamaged and $50 \%$ damaged conditions are 43 and 45 , respectively. And then these values are used in the corresponding phase space reconstruction. The reconstructed phases of the cases in Figure 8 are shown in Figure 9. As shown, the 50\% damaged phase trajectory deviates from undamaged one, and the enveloped area is bigger than the undamaged one. This phenomenon indicates that the damage occurs in the beam. To further locate the damage, the method introduced in Section 3.2 is used.

In the phase space reconstruction, we cannot find the parameter of time delay Tau using the displacement responses directly with the autocorrelation function method mentioned in Section 3.2, because the displacement responses are less than zero, which will lead the autocorrelation function $c_{\mathrm{ss}}$ always less than zero. Thus the separated dominant dynamic component, as shown in Figure 5(b), is used to determine the time delay Tau, but the response used in its following phase space reconstruction and damage locating is the maintained information with the high dynamic components which are removed.

4.3.2. Scenarios of Different Mass of the Moving Mass. The issue in this section is to evaluate the sensitivity of the proposed method to the mass of the moving mass which is set to be $m_{m}=50 \mathrm{Kg}, 200 \mathrm{Kg}$, and $400 \mathrm{Kg}$, respectively. The details of this analysis scenario are shown as the scenario 1 in
Table 1. Figure 10 shows the acceleration responses with the moving mass is $50 \mathrm{Kg}$ and $400 \mathrm{Kg}$. As shown in Figure 10(a), the damage cannot be indicated although the beam has been $50 \%$ damaged at the location for $L_{d}=0.4$, shown as the red dotted line (the same in the Figures 11-18). This phenomenon also happens in case mass is $400 \mathrm{Kg}$, shown in Figure 10(b).

Firstly, the acceleration responses are filtered with MFF and the maintained responses are reconstructed into the phase spaces. Then the proposed damage index CPST is calculated to identify the damage. Figure 11 is the results of the CPST curves of different damage levels with the mass which is $50 \mathrm{Kg}$ in analysis scenario 1 (see Table 1). As shown, in the curve of each damage level, there is a peak at the position of damage. But it is not obvious because there are many peaks as well at other positions along the length of the beam, which interfere us to make the judgement. Hence, the results are filtered again using the MFF to eliminate the interference. Figure 12 shows the filtered results related to that shown in Figure 11. As shown, the wave in the curve is removed and becomes smooth. For the $30 \%, 40 \%$, and $50 \%$ damaged scenarios, each CPST curve has a slope discontinuity at the damage location, while in $10 \%$ and $20 \%$ damaged scenarios, it is not obvious as that of high damage levels because of the perform size of this figure. If the result of low damaged level is separated to be plotted, this phenomenon still exists, shown as a result of 10\% damage scenario in Figure 13.

In order to evaluate the sensitivity of the CPST to displacement response and compare the effectiveness using the two types of response, the displacement response is also used to identify the damage. Figure 14 shows the displacement responses of the midspan point for different damage levels with the mass which is $50 \mathrm{Kg}$. As shown, the displacement increases with damage. As can be noted, although displacement increases with damage, the increase is relatively insignificant and this information cannot be used to locate the 


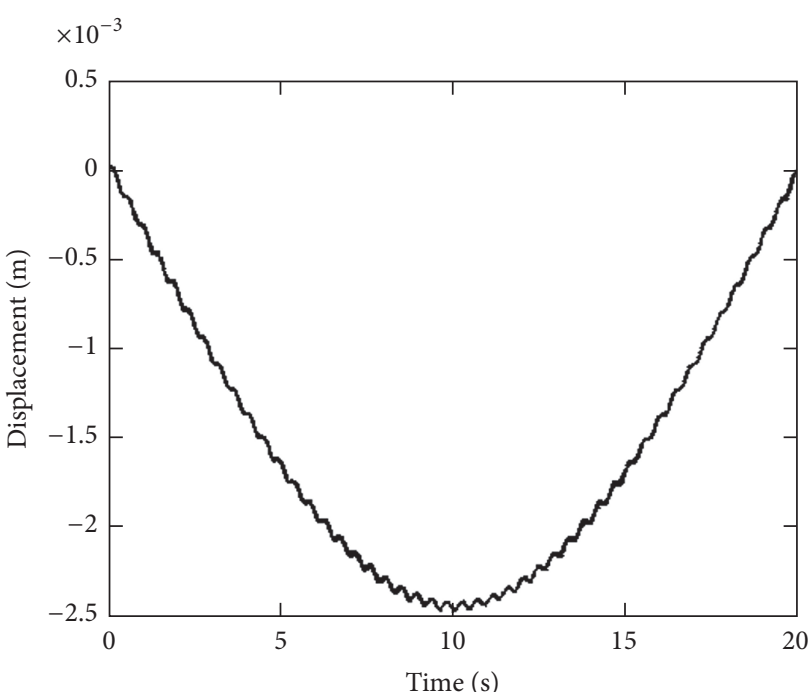

(a)

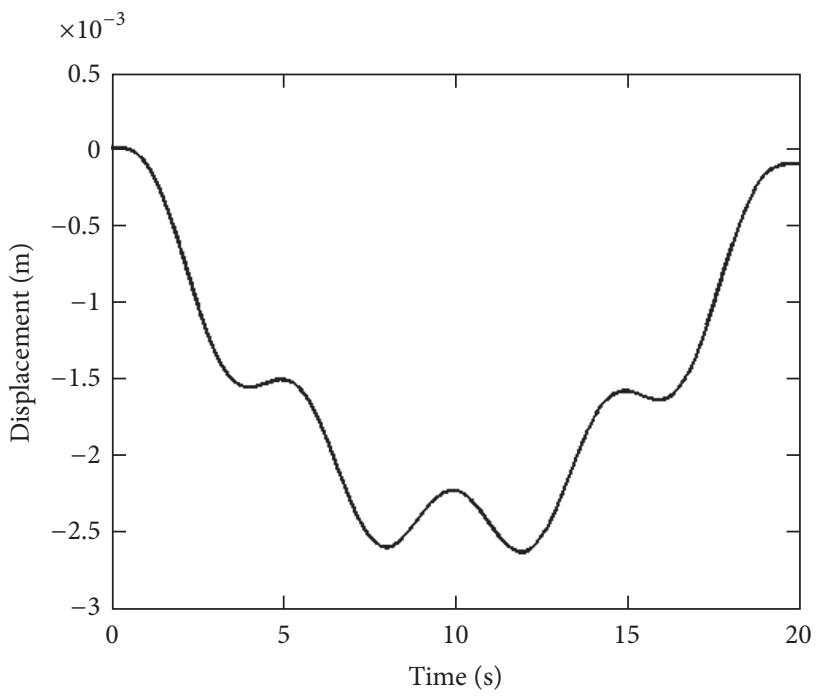

(c)

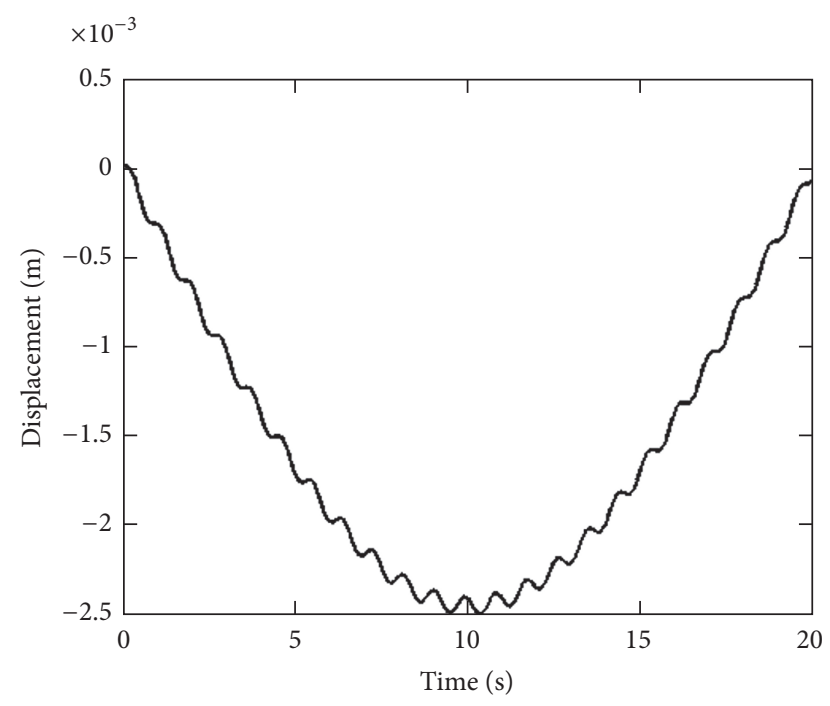

(b)

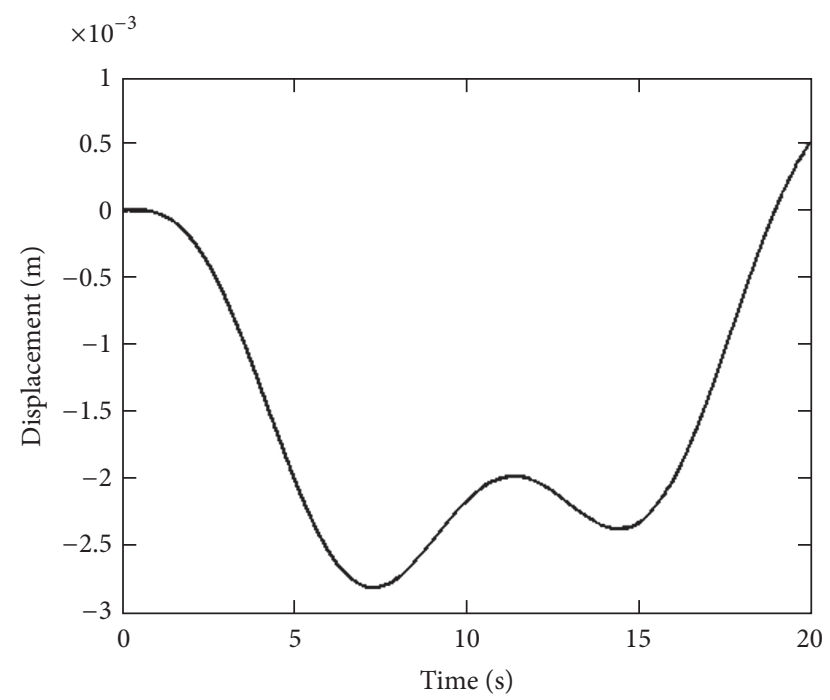

(d)

FIGURE 24: Displacement responses of different mass velocity scenarios with $m_{m}=200 \mathrm{Kg}, L_{d}=0.4$, and $L_{s}=0.5$ : (a) $v=0.5 \mathrm{~m} / \mathrm{s}$, (b) $v=1 \mathrm{~m} / \mathrm{s}$, (c) $v=5 \mathrm{~m} / \mathrm{s}$, and (d) $v=10 \mathrm{~m} / \mathrm{s}$.

damage. Using the component, which dominates the displacement responses, the time delay Tau of the maintained responses corresponding to each damage level is calculated, and then the corresponding phase space is reconstructed. The calculated CPST from the displacement dynamic responses of different damage level is shown in Figure 15. As shown, the damage locations are confidently identified with the peaks at $x / L=0.4$ when the crack depth ratio is equal to or greater than $20 \%$. The CPST value increases significantly with the damage depth ratio increase. For the 10\% damage scenario, as in acceleration analysis, the peak is relatively insignificant and it is also separated to be shown, shown in Figure 16. As shown, the peak at $x / L=0.4$ is obvious as well. It should be note that although the CPST values can indicate the increasing tendency of damage ratio, it is not able to identify the damage depth accurately. To identify the damage depth should need the model updating method, which is not the topic of this paper.

In the following analysis, keeping the mass velocity $v$, damage location $L_{d}$, and senor location $L_{s}$ at the corresponding constant value as the $10 \%$ damaged scenario in this section, the scenarios with the moving mass for $200 \mathrm{Kg}$ and $400 \mathrm{Kg}$ are simulated, respectively. The CPST results using both types of responses are calculated as shown in Figures 17 and 18 for the case with moving mass which is $200 \mathrm{Kg}$ and $400 \mathrm{Kg}$, respectively. As shown, comparing the cases with different mass, the results of both types of responses for $200 \mathrm{Kg}$ and $400 \mathrm{Kg}$ cases are similar to that of $50 \mathrm{Kg}$ case but the CPST value increases with the mass increases. The results using displacement responses are also more obvious than that of acceleration responses. This is because acceleration is the two-order derivative of displacement. When the 


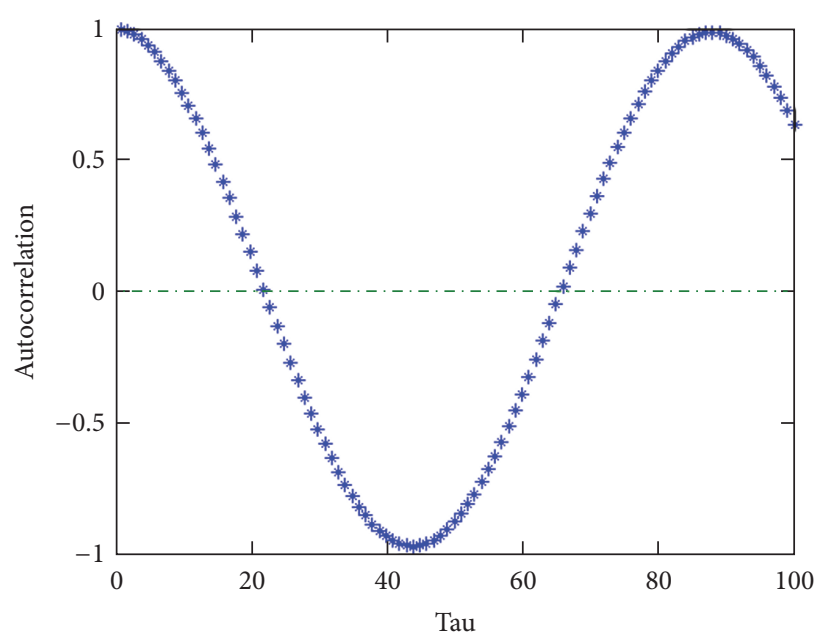

(a)

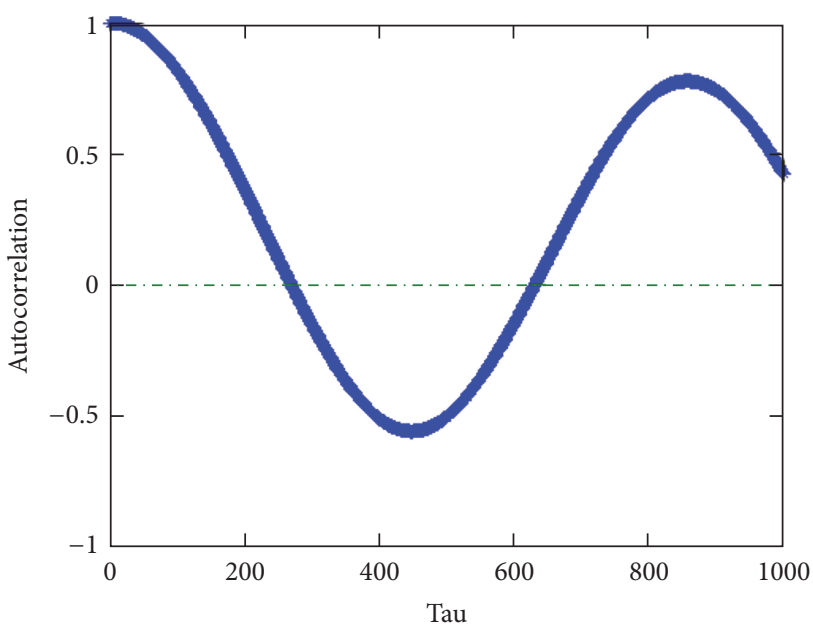

(c)

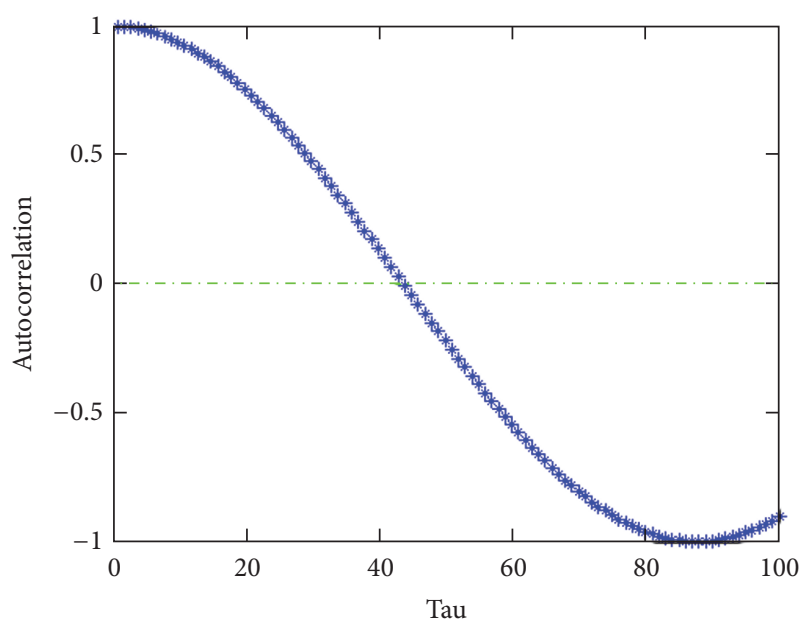

(b)

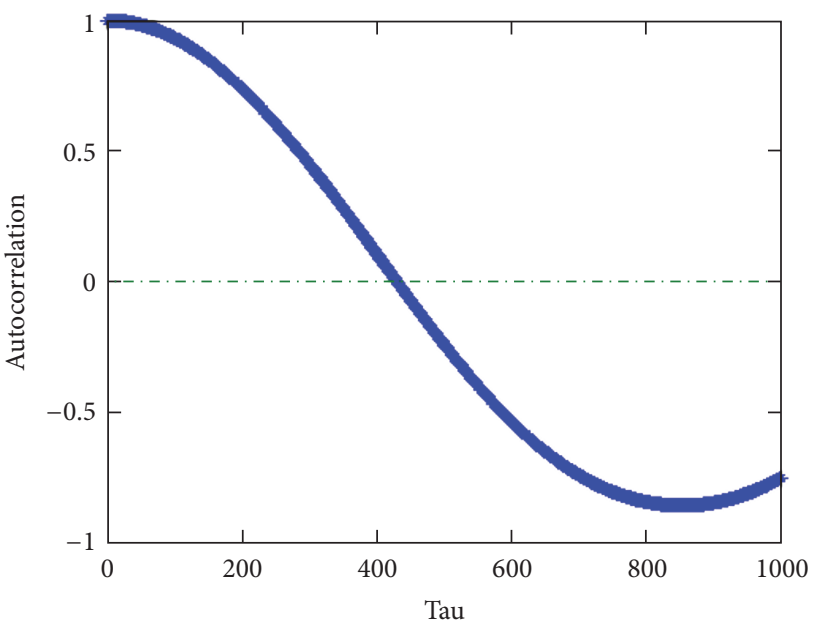

(d)

FIGURE 25: Autocorrelation of different mass velocity with damaged level is $10 \%$, mass is $200 \mathrm{Kg}$, and sensor location is $L_{s}=0.5$ : (a) mass velocity is $v=0.5 \mathrm{~m} / \mathrm{s}$, (b) mass velocity is $v=1 \mathrm{~m} / \mathrm{s}$, (c) mass velocity is $v=5 \mathrm{~m} / \mathrm{s}$, and (d) mass velocity is $v=10 \mathrm{~m} / \mathrm{s}$.

displacement increases in volatility, the acceleration may also fluctuate around zero, which will lead to the topology of the reconstructed phase space of displacement more sensitive to damage than that of acceleration. These results indicate that phase space-based method using displacement responses can effectively identify the damage of the beam and more reliable than using acceleration responses.

4.3.3. Scenarios of Different Damage Location. In order to evaluate the sensitivity of CPST to damage location, the scenario with different damage location for $L_{d}$ equal to 0.2 , 0.40 .6 , and 0.8 , others factors are kept to be a constant value, is simulated, respectively. The details of others factors are shown as the analysis scenario 2 in Table 1 . In this issue, only the $10 \%$ damaged scenario is analyzed because it is more representative than that of high damaged levels. Figure 19 shows the results using acceleration responses. The damage location is indicated by a small red circle here (as the same in the following figures). As shown, the CPST curve of each damage location case, as in the previous issue, presents a slope discontinuity.

Figure 20 shows the results using displacement responses. As shown, the CPST curves of all the cases present a significant peak at the damage location. This result indicates again that the CPST index using displacement responses is more sensitive than that of using acceleration responses. The CPST value at the peak in the case with the damage location near the ends of the beam is smaller than the cases with damage far away from the ends, such that the peak value is $0.49 e-4$ when $L_{d}=0.2$ shown in Figure $20(\mathrm{a})$, and it is $1.50 e-4$ when $L_{d}=0.4$ shown in Figure 20(b). The peak values are relatively equivalent when the damage location is symmetrical, such as the cases with $L_{d}=0.2$ and 0.8 and the cases with $L_{d}=0.4$ and 0.6 as well.

4.3.4. Scenarios of Different Sensor Location. The issue in this section is to evaluate the sensitivity of CPST to the sensor location $L_{s}$ which is set to be $L_{s}=0.25,0.50$, and 


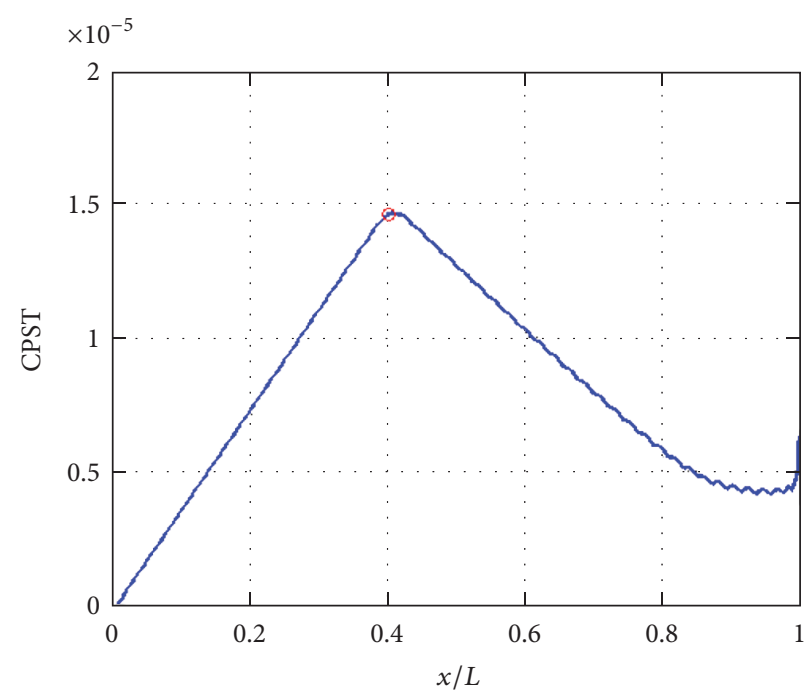

(a)

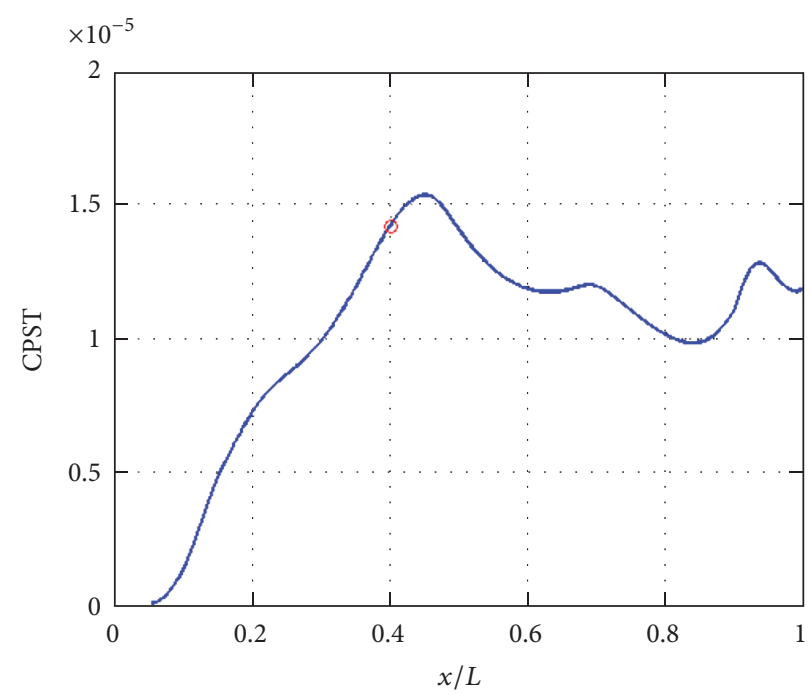

(b)

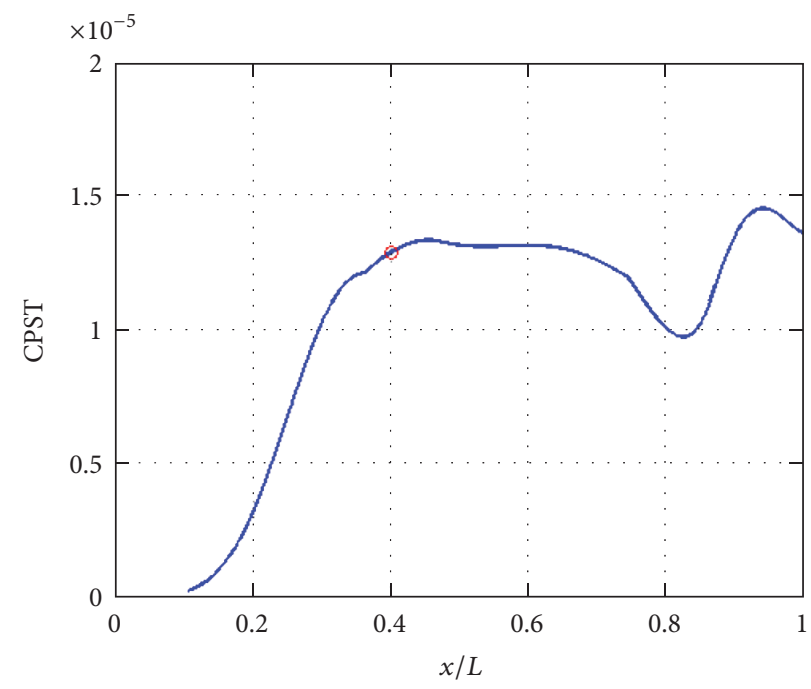

(c)

FIGURE 26: CPST results of different mass velocity with damaged level is $10 \%$, mass is $200 \mathrm{Kg}$, and sensor location is $L_{s}=0.5$ : (a) mass velocity is $v=0.5 \mathrm{~m} / \mathrm{s}$, (b) mass velocity is $v=5 \mathrm{~m} / \mathrm{s}$, and (c) mass velocity is $v=10 \mathrm{~m} / \mathrm{s}$.

0.75 , respectively. Others factors are kept to be the constant value shown as the analysis scenario 3 in Table 1 . The simulated responses measured at different location are shown in Figure 21(a) which shows displacement responses and Figure 21(b) which shows acceleration responses. As shown in Figure 21(a), the displacement at $L_{s}=0.5$ is greater than the ones $L_{s}=0.25$ and 0.75 , and the peak of the case for $L_{s}=0.25$ deviates to the left of the center, while it deviates to the right in the case for $L_{s}=0.75$. The acceleration responses, shown in Figure 21(b), do not present significant difference as displacement. These responses with the damage depth ratio for $10 \%$ are all used in the damage detection and the results are shown in Figure 22 for acceleration responses and Figure 23 for displacement responses. The acceleration response result of the case for $L_{s}=0.5$ is analyzed in Section 4.3.3 and is shown in Figure 19(b), and displacement response result of the same case is shown in Figure 20(b). As shown in Figures 22 and 19(b), the CPST curves of three cases have slope discontinuity as previous analysis scenarios. The displacement responses results shown in Figures 23 and 20(b) present a significant peak at the damage location for $L_{d}=0.4$. The peak value of the case for $L_{s}=0.5$ is large than that of the case $L_{s}$ far away from the beam center. These results indicate that the damage of the beam can be confidently identified with the sensor installed at different location, although the sensor location for $L_{s}=0.5$ is the best.

4.3.5. Scenarios of Different Moving Mass Velocity. In the previous analysis scenarios, the velocity of the moving mass is set to be a constant value for $v=1 \mathrm{~m} / \mathrm{s}$. The issue in this section is to evaluate the sensitivity of CPST to the mass velocity $v$ which is set to be $v=0.5 \mathrm{~m} / \mathrm{s}, 1 \mathrm{~m} / \mathrm{s}, 5 \mathrm{~m} / \mathrm{s}$, and $10 \mathrm{~m} / \mathrm{s}$, respectively. Other factors are also kept to be the constant value shown as the analysis scenario 4 in the Table 1. 

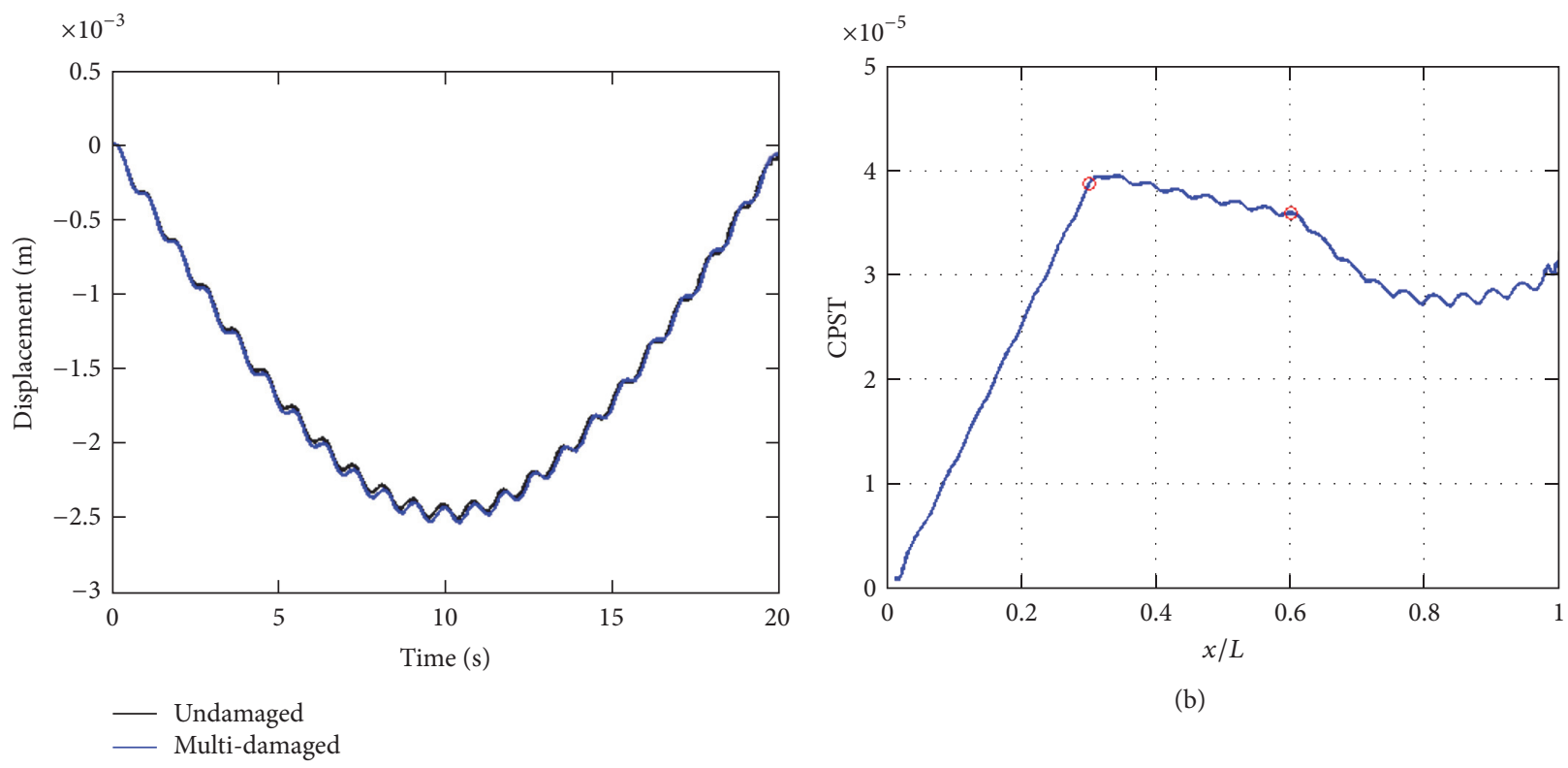

(b)

(a)

FIGURE 27: Multidamaged results: (a) is displacement responses and (b) is the CPST curve.

In this issue, only the displacement responses are analyzed to calculate the CPST.

Responses at midspan for different velocities of the moving mass are plotted in Figure 24. The figure shows how the amplitude of the dynamic response increases with the velocity. It also shows how the amplitude of the higher frequency wave increases as the velocity increases. In correspondence with the velocities, $0.5,1,5$, and $10 \mathrm{~m} / \mathrm{s}$, the number of cycles of the high frequency wave reduces from 45 to 22,4 , and 2 , respectively. This will affect the time delay Tau in phase space reconstruction process, as explained in Section 3. The autocorrelations used to determine the Tau are plotted in Figure 25. As shown, the first crossing zero point increases from 22 to 43, 269, and 453. The CPST results are shown in Figure 26 for the cases with the mass velocity for $0.5,5$, and $10 \mathrm{~m} / \mathrm{s}$, and the result for $1 \mathrm{~m} / \mathrm{s}$ case is plotted in Figure 20(b). As shown, the damage location can be detected confidently when the velocity is less than $5 \mathrm{~m} / \mathrm{s}$. With the mass velocity increasing, the results become ambiguous, such that in the $5 \mathrm{~m} / \mathrm{s}$ case, the curve peak deviates from damage location marked by the red circle. In $10 \mathrm{~m} / \mathrm{s}$ case, the figure shows the presence of spurious local maxima in the CPST curve, which leads to the fact that it fails to identify the damage location, because the reconstructed phase space needs to use the time delay method, which will lose some of information of the response. If the mass speed is higher, the waves of the response are less and more information will lose. This is the disadvantage of the proposed method.

4.3.6. Scenarios of Multiple Types of Damage. The issue in this section is to evaluate the effectiveness of CPST to multiple types of damage which are set to be $L_{d}=0.3$ and 0.6 with the damage depth ratio for $20 \%$ and $10 \%$, respectively.
Others factors are kept to be the constant value shown as the analysis scenario 5 in Table 1 . The midspan displacement responses of undamaged and multidamaged beam are shown in Figure 27(a). Comparing the two responses, as shown, there is no big difference between the two curves except for slight deviation. But the CPST curve of this scenario, shown in Figure 27(b), has two significant peaks at the locations $x / L=0.3$ and 0.6 . The two locations are just the damage locations set in the simulation. The peak value at $x / L=0.3$ is relatively larger than that at $x / L=0.6$, because the damage depth ratio at $x / L=0.3$ is $20 \%$ while $10 \%$ at $x / L=0.6$. These results indicate that the proposed method can also identify multiple types of damage in the beam.

4.4. Robustness to Noise. In SHM of civil infrastructure, with the influence of measurement noises and changing ambient loads from wind, temperature, humidity, and so forth, mode parameters might not be accurately identified using limited sensors. These in turn may result in the mode shape-based indices not able to detect structural damage [33]. To study the robustness of this approach in a noise contaminated environment, the response time histories smeared with different levels Gaussian noise are also used in the analysis. In this issue, the details of the factors are shown in the analysis scenario 6 in the Table 1.

The influence of ambient noise is considered, smearing a white noise to the displacement response $w(t)$, calculated through

$$
w_{\text {noise }}=w+N_{\text {lev }} \operatorname{RMS}(w) \eta,
$$

where $N_{\text {lev }}$ is the noise level, RMS $(w)$ is the root mean square of the displacement response, and $\eta$ is the normal distribution vector with zero means and unitary rms. Here the responses 


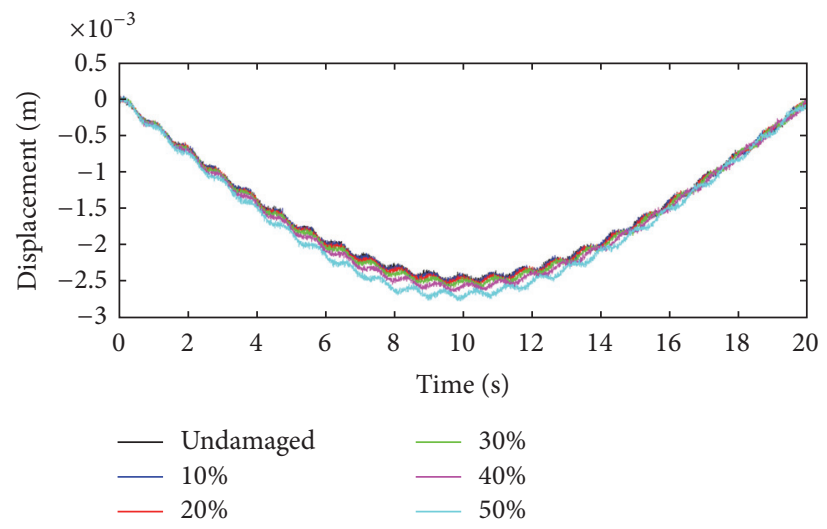

(a)

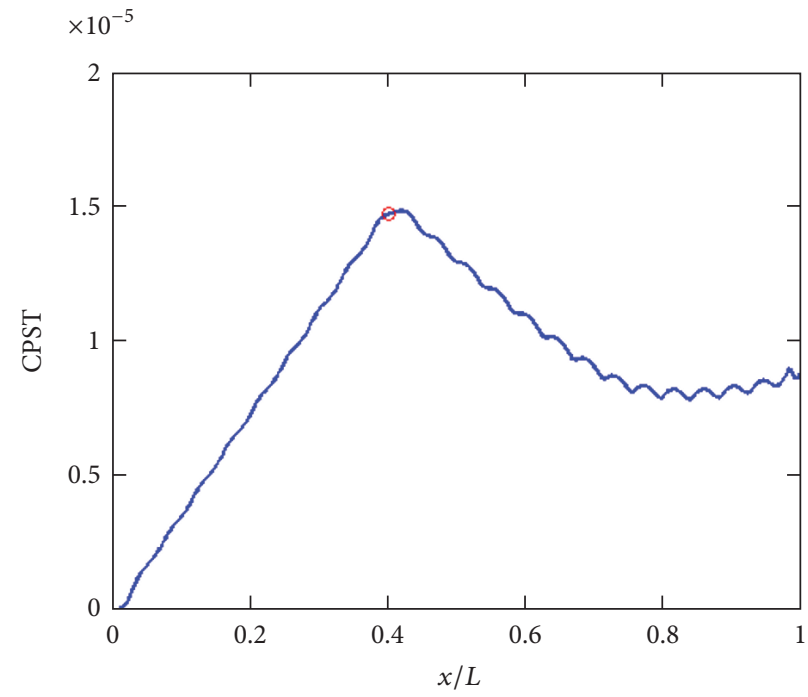

(b)

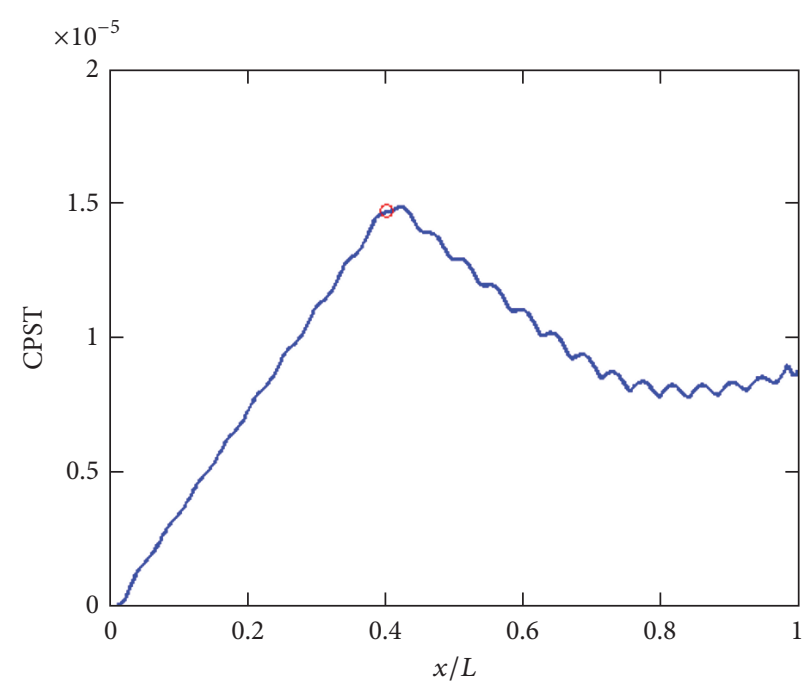

(c)

FIGURE 28: Results using noise contaminated displacement responses with $v=1 \mathrm{~m} / \mathrm{s}, m_{m}=200 \mathrm{Kg}, L_{d}=0.4$, and $L_{s}=0.5$ : (a) $30 \%$ noise contaminated displacement responses, (b) CPST for 10\% damaged scenario with $10 \%$ noise, and (c) CPST curve for $10 \%$ damaged scenario with $30 \%$ noise.

are smeared with $10 \%$ and $30 \%$ normal distributed noise. The above analysis is repeated using these noise contaminated responses.

The plot in Figure 28(a) shows the displacement responses contaminated with $10 \%$ noise. Figures $28(\mathrm{~b})$ and 28 (c) show the $10 \%$ damaged CPST curve evaluated for $10 \%$ and $30 \%$ noise level. As shown, even if the noise level is $30 \%$, the damage position can be still correctly identified from the location of the highest crest, and the results for $0 \%$ (shown in Figure 20(b)), 10\%, and 30\% noise levels are relatively the same as each other. This is because the responses are filtered by MFF, and the high dynamic components together with the noise are removed. The maintained components are almost the same, although the high noise level contaminated responses are treated by MFF twice or more times using the same $T_{k}$. These results confirm the robustness to noise and reliability of the proposed method even with heavily contaminated measurements.

\section{Conclusions}

This paper presents a novel damage detection method based on the reconstructed phase space of vibration signals using a single sensor. In this approach, a moving mass is applied as excitation source, and the structure vibration responses at different positions are measured using a single sensor. The choice of the proper delay time and embedding dimensions for phase space reconstruction are presented. A Moving Filter Function (MFF) is also presented to be used to separate and filter the responses before phase space reconstruction. Using the determined time delay and embedding dimensions, the responses are translated from time domain into the spatial domain. The index CPST values are calculated from the reconstructed phase space and used to identify structural damage. To demonstrate the method, six analysis scenarios for a beam-like structure considering the moving mass magnitude, damage location, the single sensor location, moving 
mass velocity, multiple types of damage, and the responses contaminated with noise are calculated. The acceleration and displacement responses are both used to identify the damage. The results indicate that the proposed method using displacement response is more sensitive to damage than that of acceleration responses. The results also proved that the proposed method can use a single sensor installed at different location of the beam to locate the damage/much damage reliably, even though the responses are contaminated with noise. This approach is closely related to the practical engineering, does not show the problem of optimizing the allocation of sensors, and is a qualitative leap in the sensor consumption. However, as the approach proceeding presented, it is necessary to use the undamaged response as the baseline, and the method will fail to identify the damage if the vehicle passes with a high speed. These is the limitation of the current CPST indicator, and further research is deemed necessary to overcome these shortcoming. Nonetheless, this approach can use only one sensor to identify the existence and location of damage. Therefore, CPST is a good candidate to be used in continuous online SHM to identify the damage.

\section{Competing Interests}

The authors declare that they have no competing interests.

\section{Acknowledgments}

The authors acknowledge the financial supports from the National Natural Science Foundation of China under Grant no. 11402098, the Major Special Fund of Guangdong Province under Grant no. 2012A080102008, Doctoral Fund of Ministry of Education of China (20134401120009), and International Science and Technology Cooperation Fund of Qing Hai Province (2014-HZ-822).

\section{References}

[1] Z. Nie, H. Hao, and H. Ma, "Structural damage detection based on the reconstructed phase space for reinforced concrete slab: experimental study," Journal of Sound and Vibration, vol. 332, no. 4, pp. 1061-1078, 2013.

[2] N. T. Khiem and T. V. Lien, "A simplified method for natural frequency analysis of a multiple cracked beam," Journal of Sound and Vibration, vol. 245, no. 4, pp. 737-751, 2001.

[3] O. S. Salawu, "Detection of structural damage through changes in frequency: a review," Engineering Structures, vol. 19, no. 9, pp. 718-723, 1997.

[4] N. Lieven and D. Ewins, "Spatial correlation of modal shapes, the coordinate modal assurance criterion (COMAC)," in Proceedings of the 6th International Modal Analysis Conference, vol. 1, pp. 690-695, 1988.

[5] A. K. Pandey and M. Biswas, "Damage detection in structures using changes in flexibility," Journal of Sound and Vibration, vol. 169, no. 1, pp. 3-17, 1994.

[6] A. K. Pandey, M. Biswas, and M. M. Samman, "Damage detection from changes in curvature mode shapes," Journal of Sound and Vibration, vol. 145, no. 2, pp. 321-332, 1991.

[7] K. Roy and S. Ray-Chaudhuri, "Fundamental mode shape and its derivatives in structural damage localization," Journal of Sound and Vibration, vol. 332, no. 21, pp. 5584-5593, 2013.
[8] Z. Ismail, H. Abdul Razak, and A. G. Abdul Rahman, "Determination of damage location in RC beams using mode shape derivatives," Engineering Structures, vol. 28, no. 11, pp. 15661573, 2006.

[9] R. P. Bandara, T. H. T. Chan, and D. P. Thambiratnam, "Frequency response function based damage identification using principal component analysis and pattern recognition technique," Engineering Structures, vol. 66, pp. 116-128, 2014.

[10] P. C. Chang, A. Flatau, and S. C. Liu, "Review paper: health monitoring of civil infrastructure," Structural Health Monitoring, vol. 2, no. 3, pp. 257-267, 2003.

[11] Y. J. Yan, L. Cheng, Z. Y. Wu, and L. H. Yam, "Development in vibration-based structural damage detection technique," Mechanical Systems and Signal Processing, vol. 21, no. 5, pp. 2198-2211, 2007.

[12] X. Q. Zhu and S. S. Law, "Wavelet-based crack identification of bridge beam from operational deflection time history," International Journal of Solids and Structures, vol. 43, no. 7-8, pp. 2299-2317, 2006.

[13] D. Hester and A. González, "A wavelet-based damage detection algorithm based on bridge acceleration response to a vehicle," Mechanical Systems and Signal Processing, vol. 28, pp. 145-166, 2012.

[14] K. V. Nguyen and H. T. Tran, "Multi-cracks detection of a beam-like structure based on the on-vehicle vibration signal and wavelet analysis," Journal of Sound and Vibration, vol. 329, no. 21, pp. 4455-4465, 2010.

[15] A. Khorram, F. Bakhtiari-Nejad, and M. Rezaeian, "Comparison studies between two wavelet based crack detection methods of a beam subjected to a moving load," International Journal of Engineering Science, vol. 51, pp. 204-215, 2012.

[16] A. Khorram, M. Rezaeian, and F. Bakhtiari-Nejad, "Multiple cracks detection in a beam subjected to a moving load using wavelet analysis combined with factorial design," European Journal of Mechanics. A. Solids, vol. 40, pp. 97-113, 2013.

[17] K. V. Nguyen, "Comparison studies of open and breathing crack detections of a beam-like bridge subjected to a moving vehicle," Engineering Structures, vol. 51, pp. 306-314, 2013.

[18] Y. Christos, M. Jedrzej, R. Konstantinos, and A. Ioannis, "Multicomponent decomposition of a time-varying acoustic Doppler signal generated by a passing railway vehicle using Complex Shifted Morlet Wavelets," Transportation Research Part C: Emerging Technologies, vol. 44, pp. 34-51, 2014.

[19] N. Roveri and A. Carcaterra, "Damage detection in structures under traveling loads by Hilbert-Huang transform," Mechanical Systems and Signal Processing, vol. 28, pp. 128-144, 2012.

[20] J. Li and S. S. Law, "Damage identification of a target substructure with moving load excitation," Mechanical Systems and Signal Processing, vol. 30, pp. 78-90, 2012.

[21] J. Li, S. S. Law, and H. Hao, "Improved damage identification in bridge structures subject to moving loads: numerical and experimental studies," International Journal of Mechanical Sciences, vol. 74, pp. 99-111, 2013.

[22] F. Cavadas, I. F. C. Smith, and J. Figueiras, "Damage detection using data-driven methods applied to moving-load responses," Mechanical Systems and Signal Processing, vol. 39, no. 1-2, pp. 409-425, 2013.

[23] Y. Zhang, S. T. Lie, and Z. Xiang, "Damage detection method based on operating deflection shape curvature extracted from dynamic response of a passing vehicle," Mechanical Systems and Signal Processing, vol. 35, no. 1-2, pp. 238-254, 2013. 
[24] M. D. Todd, J. M. Nichols, L. M. Pecora, and L. N. Virgin, "Vibration-based damage assessment utilizing state space geometry changes: local attractor variance ratio," Smart Materials and Structures, vol. 10, no. 5, pp. 1000-1008, 2001.

[25] J. M. Nichols, M. D. Todd, M. Seaver, and L. N. Virgin, "Use of chaotic excitation and attractor property analysis in structural health monitoring," Physical Review E, vol. 67, no. 1, part 2, Article ID 016209, 2003.

[26] J. M. Nichols, L. N. Virgin, M. D. Todd, and J. D. Nichols, "On the use of attractor dimension as a feature in structural health monitoring," Mechanical Systems and Signal Processing, vol. 17, no. 6, pp. 1305-1320, 2003.

[27] L. A. Overbey and M. D. Todd, "Analysis of local state space models for feature extraction in structural health monitoring," Structural Health Monitoring, vol. 6, no. 2, pp. 145-172, 2007.

[28] L. Moniz, J. M. Nichols, C. J. Nichols et al., "A multivariate, attractor-based approach to structural health monitoring," Journal of Sound and Vibration, vol. 283, no. 1-2, pp. 295-310, 2005.

[29] N. Zhenhua, H. Hong, and M. Hongwei, "Structural damage detection using phase space geometry changes," in Proceedings of the 4th Internation Conference on Experimental Vibration Analysis for Civil Engineering Structures (EVACS '11), pp. 407416, Varenna, Italy, 2011.

[30] J. M. Nichols, "Structural health monitoring of offshore structures using ambient excitation," Applied Ocean Research, vol. 25, no. 3, pp. 101-114, 2003.

[31] L. A. Overbey, C. C. Olson, and M. D. Todd, "A parametric investigation of state-space-based prediction error methods with stochastic excitation for structural health monitoring," Smart Materials and Structures, vol. 16, no. 5, pp. 1621-1638, 2007.

[32] Z. Nie, H. Hao, and H. Ma, "Using vibration phase space topology changes for structural damage detection," Structural Health Monitoring, vol. 11, no. 5, pp. 538-557, 2012.

[33] Z. Nie, H. Jiang, and H. Ma, "Structural damage detection using response phase space for beam-like structure under a moving load," in Proceedings of the 20th International Congress on Sound and Vibration (ICSV'13), pp. 2711-2719, Bangkok, Thailand, July 2013.

[34] M. A. Mahmoud and M. A. Abou Zaid, "Dynamic response of a beam with a crack subject to a moving mass," Journal of Sound and Vibration, vol. 256, no. 4, pp. 591-603, 2002.

[35] A. Ariaei, S. Ziaei-Rad, and M. Ghayour, "Repair of a cracked Timoshenko beam subjected to a moving mass using piezoelectric patches," International Journal of Mechanical Sciences, vol. 52, no. 8, pp. 1074-1091, 2010.

[36] Y. Pala and M. Reis, "Dynamic response of a cracked beam under a moving mass load," Journal of Engineering Mechanics, vol. 139, no. 9, pp. 1229-1238, 2013.

[37] A. Ariaei, S. Ziaei-Rad, and M. Ghayour, "Vibration analysis of beams with open and breathing cracks subjected to moving masses," Journal of Sound and Vibration, vol. 326, no. 3-5, pp. 709-724, 2009.

[38] F. Takens, "Detecting strange attractors in turbulence," in Dynamical Systems and Turbulence, D. Rand and L. S. Young, Eds., vol. 898 of Lecture Notes in Mathematics, pp. 366-381, Springer, Berlin, Germany, 1981.

[39] H. Tong, Non-Linear Time Series Analysis, Oxford University Press, Oxford, UK, 1990.

[40] J. M. Nichols and J. D. Nichols, "Attractor reconstruction for non-linear systems: a methodological note," Mathematical Biosciences, vol. 171, no. 1, pp. 21-32, 2001.
[41] S. T. Trickey, M. D. Todd, J. M. Nichols, and M. E. Seaver, "Excitation considerations for attractor property analysis in vibration-based damage detection," in Proceedings of the Smart Nondestructive Evaluation and Health Monitoring of Structural and Biological Systems II, vol. 5047 of Proceedings of SPIE, pp. 340349, San Diego, Calif, USA, March 2003. 


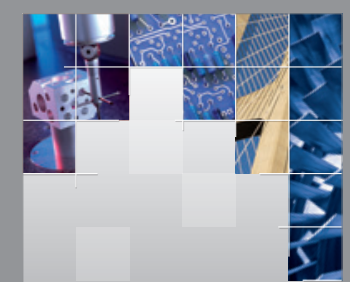

\section{Enfincering}
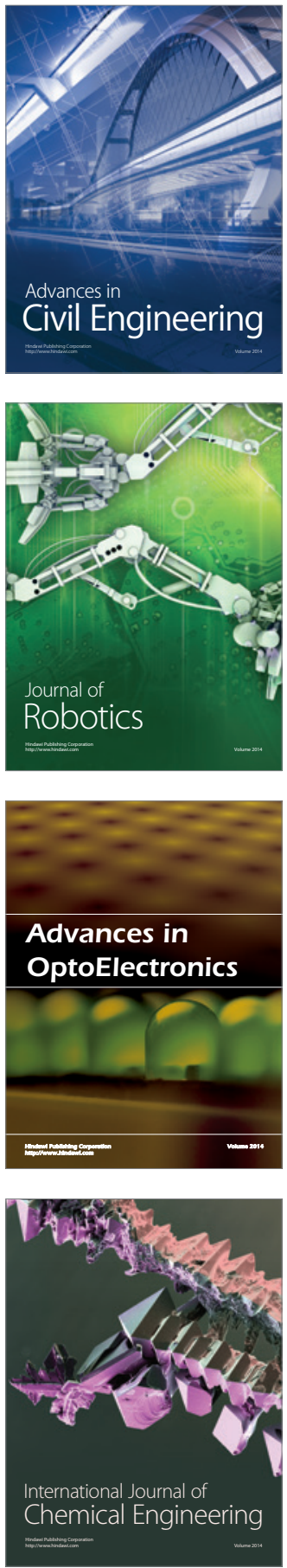

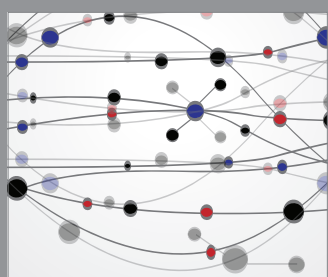

The Scientific World Journal

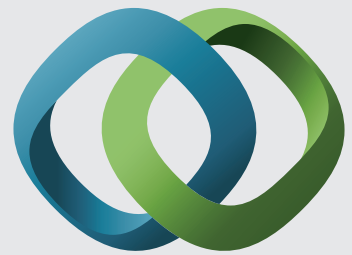

\section{Hindawi}

Submit your manuscripts at

https://www.hindawi.com
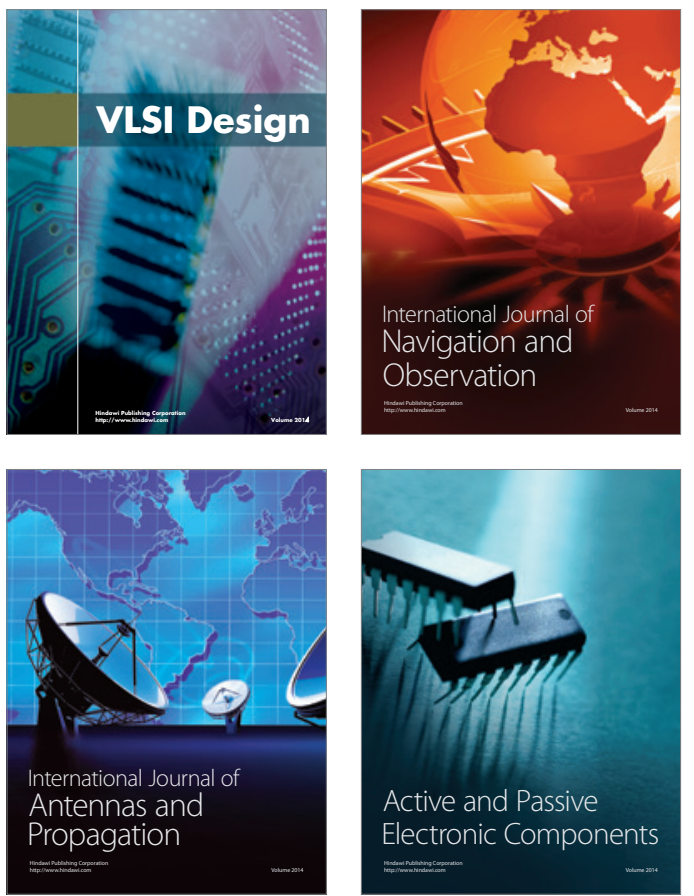
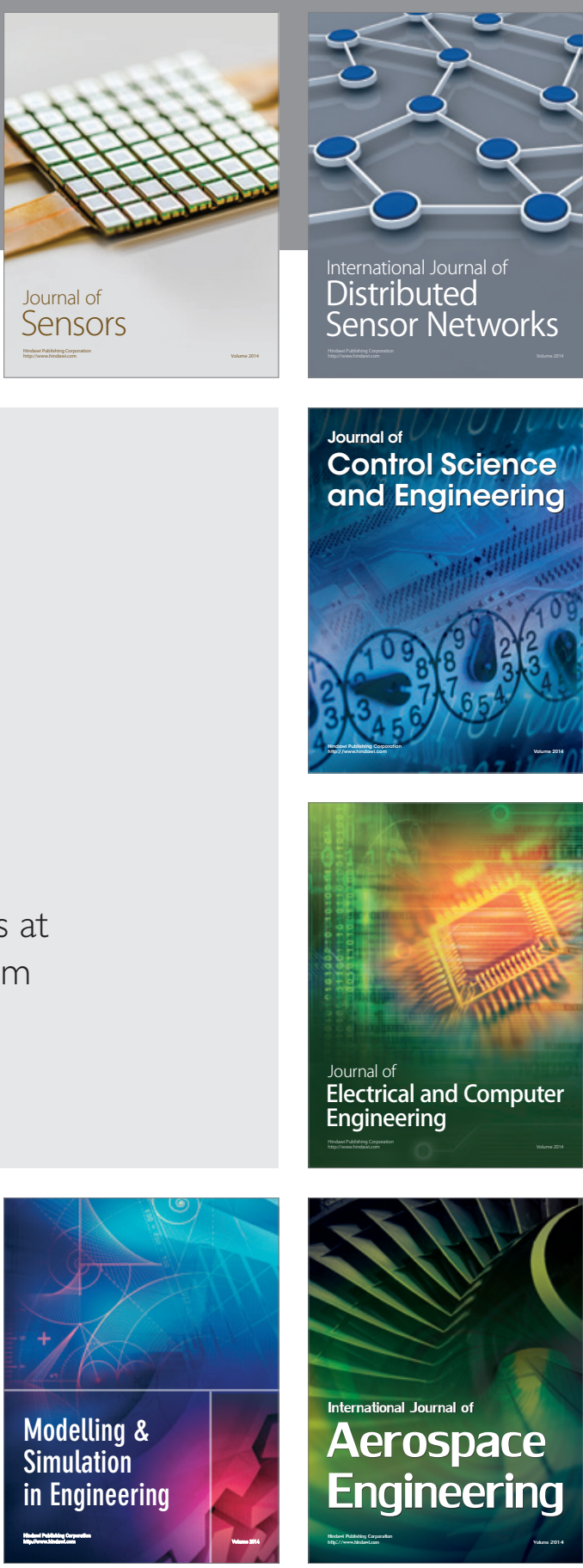

International Journal of

Distributed

Sensor Networks

$-$

Joumal of

Control Science

and Engineering
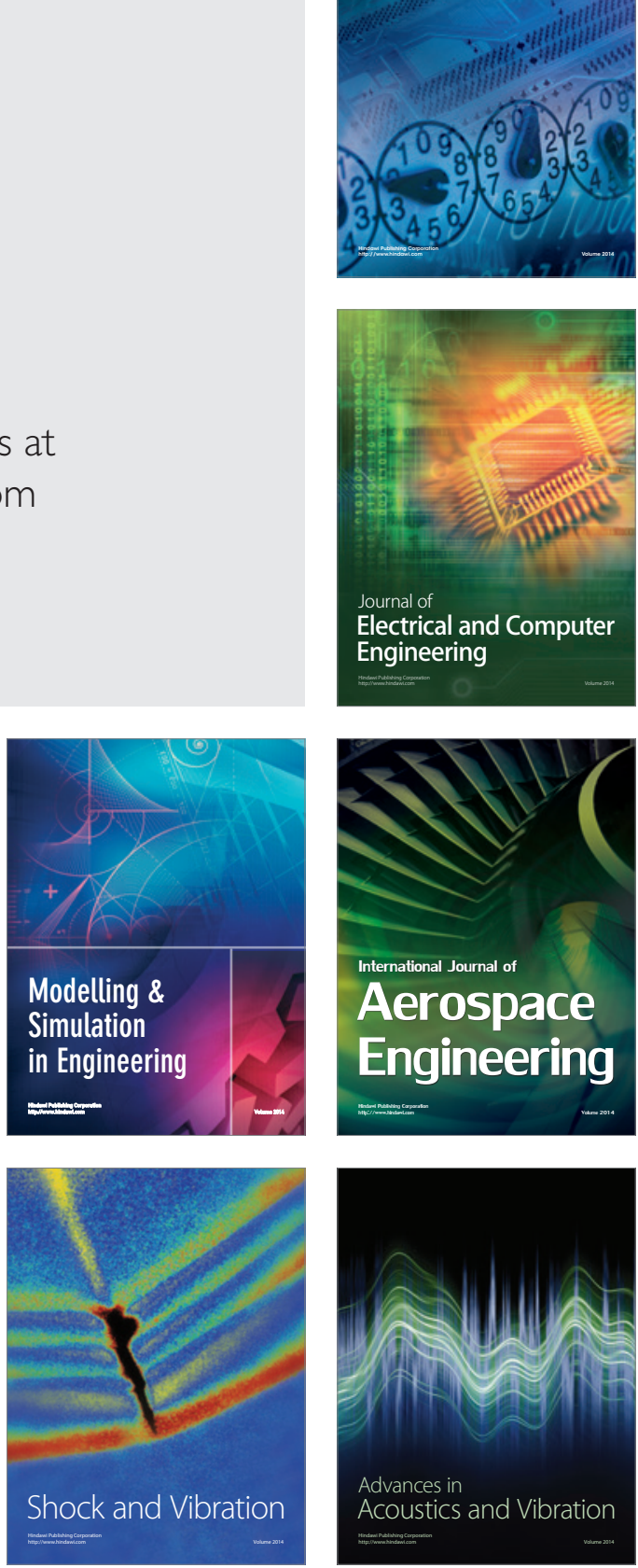